\title{
Convective instability and transient growth in flow over a backward-facing step
}

\author{
H. M. BL A CKB UR N ${ }^{1}$, \\ D. BARKLEY ${ }^{2}$ AND S. J. SHER W I N ${ }^{3}$ \\ ${ }^{1}$ Department of Mechanical and Aerospace Engineering, Monash University, \\ Victoria 3800, Australia \\ ${ }^{2}$ Mathematics Institute, University of Warwick, Coventry CV4 7AL, UK, and \\ Physique et Mécanique des Milieux Hétérogènes, Ecole Supérieure de Physique et Chimie Industrielles \\ de Paris, (PMMH UMR 7636-CNRS-ESPCI-P6-P7), 10 rue Vauquelin, 75231 Paris, France \\ ${ }^{3}$ Department of Aeronautics, Imperial College London, SW72AZ, UK
}

(Received 30 July 2007 and in revised form 13 February 2008)

Transient energy growths of two- and three-dimensional optimal linear perturbations to two-dimensional flow in a rectangular backward-facing-step geometry with expansion ratio two are presented. Reynolds numbers based on the step height and peak inflow speed are considered in the range $0-500$, which is below the value for the onset of three-dimensional asymptotic instability. As is well known, the flow has a strong local convective instability, and the maximum linear transient energy growth values computed here are of order $80 \times 10^{3}$ at $R e=500$. The critical Reynolds number below which there is no growth over any time interval is determined to be $R e=57.7$ in the two-dimensional case. The centroidal location of the energy distribution for maximum transient growth is typically downstream of all the stagnation/reattachment points of the steady base flow. Sub-optimal transient modes are also computed and discussed. A direct study of weakly nonlinear effects demonstrates that nonlinearity is stablizing at $R e=500$. The optimal three-dimensional disturbances have spanwise wavelength of order ten step heights. Though they have slightly larger growths than two-dimensional cases, they are broadly similar in character. When the inflow of the full nonlinear system is perturbed with white noise, narrowband random velocity perturbations are observed in the downstream channel at locations corresponding to maximum linear transient growth. The centre frequency of this response matches that computed from the streamwise wavelength and mean advection speed of the predicted optimal disturbance. Linkage between the response of the driven flow and the optimal disturbance is further demonstrated by a partition of response energy into velocity components.

\section{Introduction}

Flow over a backward-facing step is an important prototype for understanding the effects of separation resulting from abrupt changes of geometry in an open flow setting. The geometry is common in engineering applications and is used as an archetypical separated flow in fundamental studies of flow control (e.g. Chun \& Sung 1996), and of turbulence in separated flows (e.g. Le, Moin \& Kim 1997), which may further be linked to the assessment of turbulence models (e.g. Lien \& Leschziner 1994). The backwardfacing step geometry is also an important setting in which to understand instability of a separated flow. However, the linear instability of the basic laminar flow in such 

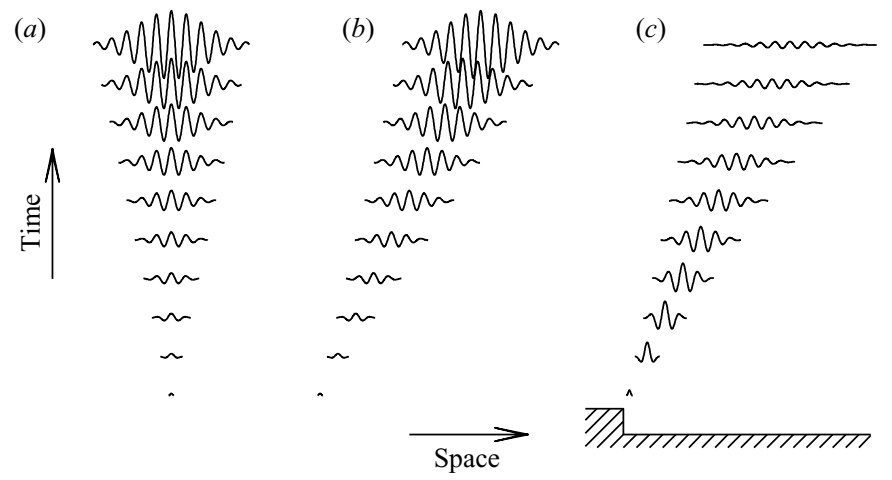

FIGURE 1. Schematic of absolute and convective instabilities. An infinitesimal perturbation, localized in space, can grow at a fixed location leading to an absolute instability $(a)$ or decay at a fixed points leading to a convective instability $(b)$. In inhomogeneous, complex geometry flow we can also observe local regions of convective instability surrounded by regions of stable flow $(c)$.

a geometry is not properly understood. While well-resolved numerical computations by Barkley, Gomes \& Henderson (2002) have determined to high accuracy both the critical Reynolds number and the associated three-dimensional bifurcating mode for the primary global instability for the case with expansion ratio of two, these results have little direct relevance to experiment. Only through careful observation has it been possible to see evidence of the intrinsic unstable three-dimensional mode (Beaudoin et al. 2004). This is because the numerical stability computations determined one type of stability threshold (of an asymptotic, or large time, global instabiliy) whereas the flow is actually unstable at much lower Reynolds numbers to a different type of instability (transient local convective instability). Moreover, the dynamics associated with the two types of instability are very different for this flow. In the present work we investigate directly the linear convective instability in this fundamental non-parallel flow by means of transient-growth computations.

To understand the issues in a broader context as well as with respect to the work presented in this paper, it is appropriate to review and contrast different concepts and approaches in (linear) hydrodynamic stability analysis. In all types of linear stability analysis one starts with a flow field $\boldsymbol{U}$, the base flow, and considers the evolution of infinitesimal perturbations $\boldsymbol{u}^{\prime}$ to the base flow. The evolution of perturbations is governed by linear equations (linearized about $\boldsymbol{U}$ ). Generally speaking, if infinitesimal perturbations grow in time, the base flow is said to be linearly unstable. However, one must distinguish between absolute and convective instability (Huerre \& Monkewitz 1985). If an infinitesimal perturbation to parallel shear flow, initially localized in space, grows at that fixed spatial location (figure 1a), then the flow is absolutely unstable. If on the other hand, the perturbation grows in magnitude but propagates as it grows such that the perturbation ultimately decays at any fixed point in space (figure $1 b$ ), then the instability is convective.

In practice, one is often interested in inhomogeneous flow geometries in which there is a local region of convective instability surrounded upstream and downstream by regions of stability (figure $1 c$ ). For illustration, we indicate a backward-facing-step geometry, but many other inhomogeneous open flows, such as bluff-body wakes, behave similarly. A localized perturbation initially grows, owing to local flow features 
near the step edge, and simultaneously advects downstream into a region of stability where the perturbation decays.

At this point, we must distinguish between different current research directions in hydrodynamic stability analysis of open flows. In one approach, arguably initiated by the work of Orr and Sommerfeld, we think primarily in terms of velocity profiles $U(y)$ (streamwise velocity as a function of the cross-stream coordinate) and analyse the stability of such profiles. The profiles may be analytic or may result from numerical computation and likewise the stability analysis may be analytic or it may contain a numerical component. Such an approach is called local analysis. In most practical cases of interest, however, the base flow is not a simple profile depending on a single coordinate. In problems in which the base flow does not vary too rapidly as a function of streamwise coordinate (i.e. $U(\epsilon x, y)$ ), we can legitimately consider a local analysis of each profile (Huerre \& Monkewitz 1990). Therefore through local sectional analysis, we can identify regions in which the flow is locally stable or unstable, and if unstable, whether the instability is locally convective or absolute. It is sometimes possible to extend these local analyses to a global analysis and even in some cases to a nonlinear analysis. However, rapid variations in flow geometry typically result in base flows which are either far from parallel or which do not vary slowly with streamwise coordinate, or both.

There is a second, largely distinct, approach to hydrodynamic stability analysis. In this approach we use fully resolved computational stability analysis of the flow field (see e.g. Barkley \& Henderson 1996). We call this direct linear stability analysis in analogy to the usage direct numerical simulation-DNS. We have the ability to fully resolve in two or even three dimensions the base flow, e.g. $\boldsymbol{U}(x, y, z)$, and to perform a global stability analysis with respect to perturbations in two or three dimensions, e.g. $\boldsymbol{u}^{\prime}=\boldsymbol{u}^{\prime}(x, y, z, t)$. We typically do not need to resort to any approximations beyond the initial linearization other than perhaps certain inflow and outflow conditions. In particular, we can consider cases with rapid streamwise variation of the flow. By postulating modal instabilities of the form: $\boldsymbol{u}^{\prime}(x, y, z, t)=\tilde{\boldsymbol{u}}(x, y, z) \exp (\lambda t)$ or in the case where the geometry has one direction of homogeneity in the $z$-direction $\boldsymbol{u}^{\prime}(x, y, z, t)=\tilde{\boldsymbol{u}}(x, y) \exp (\mathrm{i} \beta z+\lambda t)$, absolute instability analysis becomes a largescale eigenvalue problem for the global modal shape $\tilde{\boldsymbol{u}}$ and eigenvalue $\lambda$. There are algorithms and numerical techniques which allow us to obtain leading (critical or near critical) eigenvalues and eigenmodes for the resulting large problems (Tuckerman \& Barkley 2000). This approach has been found to be extremely effective for determining global instabilities in many complex geometry flows, both open and closed (Barkley \& Henderson 1996; Blackburn 2002; Sherwin \& Blackburn 2005) including weakly nonlinear stability (Henderson \& Barkley 1996; Tuckerman \& Barkley 2000).

Direct linear stability analysis has not been routinely applied to convective instabilities that commonly arise in problems with inflow and outflow conditions. One reason is that such instabilities are not typically dominated by eigenmodal behaviour, but rather by linear transient growth that can arise owing to the non-normality of the eigenmodes. A large-scale eigenvalue analysis simply cannot detect such behaviour. (However, for streamwise-periodic flow, it is possible to analyse convective instability through direct linear stability analysis, see e.g. Schatz, Barkley \& Swinney 1995.)

This brings us to a third area of hydrodynamic stability analysis known generally as non-modal stability analysis or transient growth analysis (Butler \& Farrell 1992; Trefethen et al. 1993; Schmid \& Henningson 2001; Schmid 2007). Here, the linear growth of infinitesimal perturbations is examined over a prescribed finite time interval and eigenmodal growth is not assumed. Much of the initial focus in this 
area has been on large linear transient amplification and the relationship of this to subcritical transition to turbulence in plane shear flows (Farrell 1988; Butler \& Farrell 1992). However, as illustrated in figure 1(c), the type of transient response due to local convective instability in open flows is nothing other than transient growth. While this relationship has been previously considered in the context of the Ginzberg-Landau equation (Chomaz, Huerre \& Redekopp 1990; Cossu \& Chomaz 1997; Chomaz 2005), it has not been widely exploited in the type of large-scale direct linear stability analyses that have been successful in promoting the understanding of global instabilities in complex flows. This appoach has been employed in flow over a backward-rounded step (Marquet, Sipp \& Jacquin 2006). Also, with different emphasis from the present approach, Ehrenstein \& Gallaire (2005) have directly computed modes in boundary-layer flow to analyse transient growth associated with convective instability.

This paper has two related aims. The first, more specific, aim is to accurately quantify the transient growth/convective instability in the flow over a backwardfacing-step flow with an expansion ratio of two. As stated at the outset, despite the long-standing interest in this flow, there has never been a close connection between linear stability analysis and experiments in the transition region for this flow. We shall present results that should be observable experimentally.

The second aim is more general. We are of the opinion that large-scale direct linear analysis provides the best, if not the only, route to understanding instability in geometrically complex flows. This potency has already been demonstrated for global instabilities. We believe the backward-facing-step flow studied in this paper demonstrates the ability of direct linear analysis to also capture local convective instability effects in flows with non-trivial geometry. Within the timestepperbased approach, this merely requires a change of focus from computing the eigensystem of the linearized Navier-Stokes operator to computing its singular value decomposition (Barkley, Blackburn \& Sherwin 2008). We also retain the ability to perform a full complement of time-integration-based tasks within the same code-base, in particular fully nonlinear simulations.

\section{Problem formulation}

In this section, we present the equations of interest, but largely avoid issues of numerical implementation until §3. Since the numerical approach we use is based on a primitive variables formulation of the Navier-Stokes equations, with inflow and outflow boundary conditions, our exposition is directed towards this formulation. Apart from such details, the mathematical description of the optimal growth problem found here follows almost directly from the treatments given by Corbett \& Bottaro (2000), Luchini (2000) and Hœpffner, Brandt \& Henningson (2005). The objective is to compute the energy growth of an optimal linear disturbance to a flow over a given time interval $\tau$.

\subsection{Geometry and governing equations}

Figure 2 illustrates our flow geometry and coordinate system. From an inlet channel of height $h$, a fully developed parabolic Poiseuille flow encounters a backward-facing step of height $h$. The geometric expansion ratio between the upstream and downstream channels is therefore two. We choose to fix the origin of our coordinate system at the step edge. The geometry is assumed homogeneous (infinite) in the spanwise direction. The other geometrical parameters, namely the inflow and outflow lengths, $L_{i}$ and $L_{o}$, 


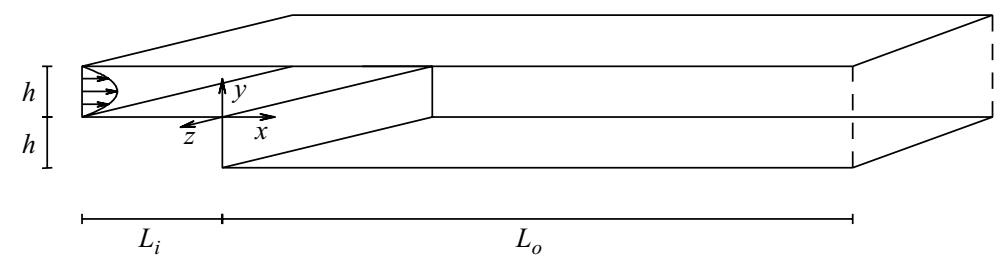

Figure 2. Flow geometry for the backward-facing step. The origin of the coordinate system is at the step edge. The expansion ratio is two. The inflow and outflow lengths, $L_{i}$ and $L_{o}$, are not to scale.

are set to ensure that the numerical solutions are independent of these parameters. As part of our convergence study in $\S 3.4$ we have found acceptable values to be $L_{i}=10 \mathrm{~h}$ and $L_{o}=50 h$ for the range of flow conditions considered.

We work in units of the step height $h$ and centreline velocity $U_{\infty}$ of the incoming parabolic flow profile. This defines the Reynolds number as

$$
R e \equiv U_{\infty} h / v,
$$

where $v$ is the kinematic viscosity, and means that the time scale is $h / U_{\infty}$.

The fluid motion is governed by the incompressible Navier-Stokes equations, written in non-dimensional form as

$$
\begin{aligned}
\partial_{t} \boldsymbol{u} & =-(\boldsymbol{u} \cdot \nabla) \boldsymbol{u}-\nabla p+R e^{-1} \nabla^{2} \boldsymbol{u} \quad \text { in } \Omega, \\
\nabla \cdot \boldsymbol{u} & =0 \quad \text { in } \Omega,
\end{aligned}
$$

where $\boldsymbol{u}(\boldsymbol{x}, t)=(u, v, w)(x, y, z, t)$ is the velocity field, $p(\boldsymbol{x}, t)$ is the kinematic (or modified) pressure field and $\Omega$ is the flow domain, such as illustrated in figure 2 . In the present work, all numerical computations will exploit the homogeneity in $z$ and require only a two-dimensional computational domain.

The boundary conditions are imposed on (2.1) as follows. First, we decompose the domain boundary as $\partial \Omega=\partial \Omega_{i} \cup \partial \Omega_{w} \cup \partial \Omega_{o}$, where $\partial \Omega_{i}$ is the inflow boundary, $\partial \Omega_{w}$ is the solid walls, i.e. the step edge and channel walls, and $\partial \Omega_{o}$ is the outflow boundary. At the inflow boundary we impose a parabolic profile, at solid walls we impose no-slip conditions, and at the downstream outflow boundary we impose a zero traction outflow boundary condition for velocity and pressure. Collectively, the boundary conditions are thus

$$
\begin{aligned}
\boldsymbol{u}(\boldsymbol{x}, t) & =\left(4 y-4 y^{2}, 0,0\right) \quad \text { for } \boldsymbol{x} \in \partial \Omega_{i}, \\
\boldsymbol{u}(\boldsymbol{x}, t) & =(0,0,0) \quad \text { for } \boldsymbol{x} \in \partial \Omega_{w}, \\
\partial_{x} \boldsymbol{u}(\boldsymbol{x}, t) & =(0,0,0), \quad p(\boldsymbol{x}, t)=0 \quad \text { for } \boldsymbol{x} \in \partial \Omega_{o} .
\end{aligned}
$$

\subsection{Base flows and linear perturbations}

The base flows for this problem are two-dimensional time-independent flows $\boldsymbol{U}(x, y)=$ $(U(x, y), V(x, y))$, therefore $\boldsymbol{U}$ obeys the steady Navier-Stokes equations

$$
\begin{aligned}
\mathbf{0} & =-(\boldsymbol{U} \cdot \nabla) \boldsymbol{U}-\nabla P+R e^{-1} \nabla^{2} \boldsymbol{U} \text { in } \Omega, \\
\nabla \cdot \boldsymbol{U} & =0 \quad \text { in } \Omega,
\end{aligned}
$$

where $P$ is defined in the associated base-flow pressure. These equations are subject to boundary conditions (2.2)

Our interest is in the evolution of infinitesimal perturbations $\boldsymbol{u}^{\prime}$ to the base flows. The linearized Navier-Stokes equations governing these perturbations are found by 
substituting $\boldsymbol{u}=\boldsymbol{U}+\epsilon \boldsymbol{u}^{\prime}$ and $p=P+\epsilon p^{\prime}$, where $p^{\prime}$ is the pressure perturbation, into the Navier-Stokes equations and keeping the lowest-order (linear) terms in $\epsilon$. The resulting equations are

$$
\begin{aligned}
\partial_{t} \boldsymbol{u}^{\prime} & =-(\boldsymbol{U} \cdot \nabla) \boldsymbol{u}^{\prime}-\left(\boldsymbol{u}^{\prime} \cdot \nabla\right) \boldsymbol{U}-\nabla p^{\prime}+R e^{-1} \nabla^{2} \boldsymbol{u}^{\prime} \quad \text { in } \Omega, \\
\nabla \cdot \boldsymbol{u}^{\prime} & =0 \quad \text { in } \Omega .
\end{aligned}
$$

These equations are to be solved subject to appropriate initial and boundary conditions as follows. The initial condition is an arbitrary incompressible flow which we denote by $\boldsymbol{u}_{0}$, i.e.

$$
\boldsymbol{u}^{\prime}(\boldsymbol{x}, t=0)=\boldsymbol{u}_{0}(\boldsymbol{x}) .
$$

The boundary conditions for (2.4) require more discussion because these relate to an important issue particular to the transient energy growth problem in an open-flow problem with outflow boundary conditions. There are actually two related issues: boundary conditions and domain size. Consider first a standard global linear stability analysis of this system. We would determine appropriate boundary conditions on the perturbation equations (2.4) by requiring that the total fields, $\boldsymbol{u}=\boldsymbol{U}+\epsilon \boldsymbol{u}^{\prime}$ and $p=P+\epsilon p^{\prime}$, satisfy boundary conditions (2.2). This leads to Neumann-type boundary conditions on the perturbation field at the outflow, as in $(2.2 c)$. Just as for the base flow, such boundary conditions for global stability computation lead to a useful approximation to the problem with an infinitely long outflow length. It is well established that eigenmodes may have significant amplitude at outflow boundaries and yet the mode structure and corresponding eigenvalue are well converged with respect to domain outflow length when employing Neumann-type boundary conditions (see e.g. Barkley 2005).

However, for transient growth computations it is not appropriate for perturbation fields to have significant amplitude at the outflow boundary. If a perturbation reaches the outflow boundary with non-negligible amplitude it is thereafter washed out of the computational domain and the corresponding perturbation energy is lost to the computation. As a result, in transient growth problems, computational domains must be of sufficient size that all perturbation fields of interest reach the outflow boundary with negligible amplitude. In practice, the computational domain must have both a longer inflow length and outflow length for a transient growth computation than for a standard stability computation.

The boundary conditions we consider for the perturbation equations (2.4) are simply homogeneous Dirichlet on all boundaries:

$$
\boldsymbol{u}^{\prime}(\partial \Omega, t)=\mathbf{0} .
$$

Such homogeneous Dirichlet boundary conditions have the benefit of simplifying the treatment of the adjoint problem because they lead to homogeneous Dirichlet boundary conditions on the adjoint fields.

\subsection{Optimal perturbations}

As stated at the outset, our primary interest is in the energy growth of perturbations over a time interval $\tau$, a parameter to be varied in our study. The energy of perturbation field at time $\tau$ relative to its initial energy is given by

$$
\frac{E(\tau)}{E(0)}=\frac{\left(\boldsymbol{u}^{\prime}(\tau), \boldsymbol{u}^{\prime}(\tau)\right)}{\left(\boldsymbol{u}^{\prime}(0), \boldsymbol{u}^{\prime}(0)\right)},
$$


where the inner product is defined as

$$
\left(\boldsymbol{u}^{\prime}, \boldsymbol{u}^{\prime}\right)=\int_{\Omega} \boldsymbol{u}^{\prime} \cdot \boldsymbol{u}^{\prime} \mathrm{d} v
$$

Letting $G(\tau)$ denote the maximum energy growth obtainable at time $\tau$ over all possible initial conditions $\boldsymbol{u}^{\prime}(0)$, we have

$$
G(\tau) \equiv \max _{\boldsymbol{u}^{\prime}(0)} \frac{E(\tau)}{E(0)}=\max _{\boldsymbol{u}^{\prime}(0)} \frac{\left(\boldsymbol{u}^{\prime}(\tau), \boldsymbol{u}^{\prime}(\tau)\right)}{\left(\boldsymbol{u}^{\prime}(0), \boldsymbol{u}^{\prime}(0)\right)} .
$$

Obtaining the maximizing energy growth over all initial conditions can be shown to be equivalent to finding the maximum eigenvalue of an auxiliary problem. The eigenvalue problem is constructed by introducing the evolution operator, $\mathscr{A}(\tau)$, which evolves the initial condition $\boldsymbol{u}^{\prime}(0)$ to time $\tau$ such that $\boldsymbol{u}^{\prime}(\tau)=\mathscr{A}(\tau) \boldsymbol{u}^{\prime}(0)$, so that

$$
\begin{aligned}
G(\tau) & =\max _{\boldsymbol{u}^{\prime}(0)} \frac{\left(\boldsymbol{u}^{\prime}(\tau), \boldsymbol{u}^{\prime}(\tau)\right)}{\left(\boldsymbol{u}^{\prime}(0), \boldsymbol{u}^{\prime}(0)\right)}, \\
& =\max _{\boldsymbol{u}^{\prime}(0)} \frac{\left(\mathscr{A}(\tau) \boldsymbol{u}^{\prime}(0), \mathscr{A}(\tau) \boldsymbol{u}^{\prime}(0)\right)}{\left(\boldsymbol{u}^{\prime}(0), \boldsymbol{u}^{\prime}(0)\right)} .
\end{aligned}
$$

If we now introduce the adjoint evolution operator, $\mathscr{A}^{*}(\tau)$, which will be discussed in the next section, then

$$
G(\tau)=\max _{\boldsymbol{u}^{\prime}(0)} \frac{\left(\boldsymbol{u}^{\prime}(0), \mathscr{A}^{*}(\tau) \mathscr{A}(\tau) \boldsymbol{u}^{\prime}(0)\right)}{\left(\boldsymbol{u}^{\prime}(0), \boldsymbol{u}^{\prime}(0)\right)} .
$$

Therefore, $G(\tau)$ is given by the largest eigenvalue of the operator $\mathscr{A}^{*}(\tau) \mathscr{A}(\tau)$. The eigenvalue is necessarily real since $\mathscr{A}^{*}(\tau) \mathscr{A}(\tau)$ is self-adjoint.

\subsection{Adjoints}

To compute efficiently the optimal energy growth, $G(\tau)$, of perturbations in the linearized Navier-Stokes equations (2.4), we must consider the adjoint system and its evolution operator $\mathscr{A}^{*}(\tau)$.

We first reformulate the linearized Navier-Stokes equations by defining $\boldsymbol{q}$ as

$$
\boldsymbol{q}=\left(\begin{array}{c}
\boldsymbol{u}^{\prime} \\
p^{\prime}
\end{array}\right),
$$

where, as previously defined, $\boldsymbol{u}^{\prime}$ is the perturbation velocity field and $p^{\prime}$ the perturbation pressure field. We must specify not only the spatial domain $\Omega$, but also the time interval of interest. Thus we define $\Gamma=\Omega \times(0, \tau)$, where the final time $\tau$ is an arbitrary positive parameter. The linearized Navier-Stokes equations together with their boundary and initial conditions can then be written compactly as

$$
\begin{aligned}
\mathscr{H} \boldsymbol{q} & =\mathbf{0} \quad \text { for }(\boldsymbol{x}, t) \in \Gamma, \\
\boldsymbol{u}^{\prime}(\boldsymbol{x}, 0) & =\boldsymbol{u}_{0}(\boldsymbol{x}), \\
\boldsymbol{u}^{\prime}(\partial \Omega, t) & =\mathbf{0},
\end{aligned}
$$

where

$$
\mathscr{H} \boldsymbol{q}=\left[\begin{array}{c|c}
-\partial_{t}-\mathrm{DN}+R e^{-1} \nabla^{2} \mid & -\nabla \\
\hline \nabla \cdot & 0
\end{array}\right]\left(\begin{array}{c}
\boldsymbol{u}^{\prime} \\
\hline p^{\prime}
\end{array}\right),
$$


with

$$
\mathrm{DN} \boldsymbol{u}^{\prime}=(\boldsymbol{U} \cdot \nabla) \boldsymbol{u}^{\prime}+(\nabla \boldsymbol{U}) \cdot \boldsymbol{u}^{\prime} .
$$

We refer to (2.7) as the forward system. The solution to this system of equations over time interval $\tau$ defines an evolution operator for the perturbation flow

$$
\boldsymbol{u}^{\prime}(\boldsymbol{x}, \tau)=\mathscr{A}(\tau) \boldsymbol{u}^{\prime}(\boldsymbol{x}, 0)=\mathscr{A}(\tau) \boldsymbol{u}_{0}(\boldsymbol{x})
$$

We analogously denote the adjoint variables by

$$
\boldsymbol{q}^{*}=\left(\begin{array}{c}
\boldsymbol{u}^{*} \\
p^{*}
\end{array}\right)
$$

where $\boldsymbol{u}^{*}$ and $p^{*}$ denote the velocity and pressure fields of the adjoint perturbation problem. The operator * does not denote complex conjugate, since all velocity and pressure fields are real-valued in the primitive variable approach considered here. The adjoint equation satisfied by $\boldsymbol{u}^{*}$ and $p^{*}$ is defined with respect to an inner product over the space-time domain $\Gamma$ and including both velocity and pressure variables

$$
\left\langle\boldsymbol{q}, \boldsymbol{q}^{*}\right\rangle=\int_{0}^{\tau} \mathrm{d} t \int_{\Omega} \boldsymbol{q} \cdot \boldsymbol{q}^{*} \mathrm{~d} v .
$$

As we shall demonstrate shortly, the space-time inner product (2.9) leads to the energy inner product result which was applied in (2.6).

Note that because we work with primitive variables, the inner products (2.5) and (2.9) do not require weight functions which are often required when using derived variables.

Under inner product (2.9), the adjoint linearized Navier-Stokes equations are

$$
\begin{array}{r}
\mathscr{H}^{*} \boldsymbol{q}^{*}=\mathbf{0} \quad(\boldsymbol{x}, t) \in \Gamma, \\
\boldsymbol{u}^{*}(\boldsymbol{x}, \tau)=\boldsymbol{u}_{\tau}^{*}(\boldsymbol{x}), \\
\boldsymbol{u}^{*}(\partial \Omega, t)=\mathbf{0}
\end{array}
$$

where

$$
\mathscr{H}^{*} \boldsymbol{q}^{*}=\left[\begin{array}{c|c}
\partial_{t}-\mathrm{DN}^{*}+R e^{-1} \nabla^{2} \mid & -\nabla \\
\hline \nabla \cdot & 0
\end{array}\right]\left(\begin{array}{c}
\boldsymbol{u}^{*} \\
\hline p^{*}
\end{array}\right),
$$

with

$$
\mathrm{DN}^{*} \boldsymbol{u}^{*}=-(\boldsymbol{U} \cdot \nabla) \boldsymbol{u}^{*}+(\nabla \boldsymbol{U})^{\mathrm{T}} \cdot \boldsymbol{u}^{*}
$$

Comparing $(2.7 d)$ with $(2.10 d)$ we observe that in the adjoint system, the $\partial_{t}$ and $(\boldsymbol{U} \cdot \nabla) \boldsymbol{u}^{*}$ terms are negated. The sign on the $\partial_{t}$ term implies that the adjoint system is only well-posed in the negative time direction. As a consequence, the initial condition $\boldsymbol{u}_{\tau}^{*}$ is specified at time $\tau$. The solution to the adjoint system (2.10) defines an evolution operator for the adjoint field which we have previously denoted as

$$
\boldsymbol{u}^{*}(\boldsymbol{x}, 0)=\mathscr{A}^{*}(\tau) \boldsymbol{u}^{*}(\boldsymbol{x}, \tau)
$$

The relationship between the evolution operators $\mathscr{A}(\tau)$ and $\mathscr{A}^{*}(\tau)$ under the spatial inner product (2.5) follows from the relationship between $\mathscr{H}$ and $\mathscr{H}^{*}$ under the spacetime inner product (2.9). For $\boldsymbol{q}$ and $\boldsymbol{q}^{*}$ satisfying the forward and adjoint systems, (2.7) and (2.10) respectively, we have:

$$
\left\langle\mathscr{H} \boldsymbol{q}, \boldsymbol{q}^{*}\right\rangle-\left\langle\boldsymbol{q}, \mathscr{H}^{*} \boldsymbol{q}^{*}\right\rangle=0 .
$$


Because we consider homogeneous boundary conditions for both the forward and adjoint linear systems, but inhomogeneous initial conditions on both problems, the only terms on the left-hand side of (2.12) to survive integration by parts are those involving time derivatives. Hence,

$$
\int_{0}^{\tau} \int_{\Omega}\left(\partial_{t} \boldsymbol{u}^{\prime}\right) \cdot \boldsymbol{u}^{*} \mathrm{~d} v \mathrm{~d} t+\int_{0}^{\tau} \int_{\Omega} \boldsymbol{u}^{\prime} \cdot\left(\partial_{t} \boldsymbol{u}^{*}\right) \mathrm{d} v \mathrm{~d} t=0
$$

or

$$
\int_{\Omega} \int_{0}^{\tau} \partial_{t}\left(\boldsymbol{u}^{\prime} \cdot \boldsymbol{u}^{*}\right) \mathrm{d} t \mathrm{~d} v=\int_{\Omega}\left[\boldsymbol{u}^{\prime} \cdot \boldsymbol{u}^{*}\right]_{0}^{\tau} \mathrm{d} v=0 .
$$

Thus, we have

$$
\left(\boldsymbol{u}^{\prime}(\tau), \boldsymbol{u}^{*}(\tau)\right)=\left(\boldsymbol{u}^{\prime}(0), \boldsymbol{u}^{*}(0)\right)
$$

It follows from this that $\mathscr{A}^{*}(\tau)$ is the adjoint of $\mathscr{A}(\tau)$ under inner product $(2.5)$, since

$$
\begin{aligned}
& \left(\boldsymbol{u}^{\prime}(\tau), \boldsymbol{u}^{*}(\tau)\right)=\left(\mathscr{A}(\tau) \boldsymbol{u}^{\prime}(0), \boldsymbol{u}^{*}(\tau)\right), \\
& \left(\boldsymbol{u}^{\prime}(0), \boldsymbol{u}^{*}(0)\right)=\left(\boldsymbol{u}^{\prime}(0), \mathscr{A}^{*}(\tau) \boldsymbol{u}^{*}(\tau)\right),
\end{aligned}
$$

so that

$$
\left(\mathscr{A}(\tau) \boldsymbol{u}^{\prime}(0), \boldsymbol{u}^{*}(\tau)\right)=\left(\boldsymbol{u}^{\prime}(0), \mathscr{A}^{*}(\tau) \boldsymbol{u}^{*}(\tau)\right) .
$$

This relationship between the forward and adjoint solutions is important in deriving (2.6). It should be stressed that this follows simply from the homogeneous boundary conditions and adjoint boundary conditions imposed on $\boldsymbol{u}^{\prime}$ and $\boldsymbol{u}^{*}$, respectively.

Finally, to compute the optimal growth of perturbations, (2.6), we must act with the operator $\mathscr{A}^{*}(\tau) \mathscr{A}(\tau)$. This is accomplished by fixing a relationship between the forward solution and the adjoint solution at time $\tau$. Specifically, we set the initial condition for the adjoint problem to be the solution to the forward problem at time $\tau$ :

$$
\boldsymbol{u}^{*}(\tau)=\boldsymbol{u}^{\prime}(\tau)
$$

Thus, the action of the operator $\mathscr{A}^{*}(\tau) \mathscr{A}(\tau)$ on a field $\boldsymbol{u}^{\prime}$ consists of evolving an initial condition $\tau$ time units under the forward system, followed immediately by evolving the result backward $\tau$ time units under the adjoint system.

\subsection{Three-dimensional perturbations}

The final issue to highlight is the treatment of three-dimensional perturbations. Since the flow domain is homogeneous in the spanwise direction $z$, we can consider a Fourier modal expansion in $z$

$$
\boldsymbol{u}^{\prime}(x, y, z)=\hat{\boldsymbol{u}}(x, y) \exp (\mathrm{i} \beta z)+\text { c.c. },
$$

where $\beta$ is the spanwise wavenumber. Linear systems (2.7) and (2.10) do not couple modes of different wavenumber, so modes for any $\beta$ can be computed independently. Since the base flow is such that $W(x, y)=0$, we may choose to work only with symmetric Fourier modes of the form

$$
\begin{aligned}
u^{\prime}(x, y, z) & =\hat{u}(x, y) \cos (\beta z), \\
v^{\prime}(x, y, z) & =\hat{v}(x, y) \cos (\beta z), \\
w^{\prime}(x, y, z) & =\hat{w}(x, y) \sin (\beta z), \\
p^{\prime}(x, y, z) & =\hat{p}(x, y) \cos (\beta z) .
\end{aligned}
$$


Such subspaces are invariant under (2.7) and (2.10), and any eigenmode of $\mathscr{A}^{*}(\tau) \mathscr{A}(\tau)$ must be of the form (2.14), or able to be constructed by linear combination of these shapes. All $z$-derivatives appearing in (2.7) and (2.10) become multiplications by either $\mathrm{i} \beta$ or $-\mathrm{i} \beta$, with the (imposed) wavenumber $\beta$ parameterizing the mode. Decomposition (2.14) is used in practice because it is more computationally efficient than a full Fourier decomposition. An additional benefit is the elimination of eigenvalue multiplicity which exists for a full set of Fourier modes.

\subsection{Summary}

The computational problem to be addressed starts with the solution of (2.3) for the base flows. These flows depend only on the Reynolds number. Then for each base flow we compute $G(\tau)$ by determining the maximum eigenvalue of $\mathscr{A}^{*}(\tau) \mathscr{A}(\tau)$. As discussed in $\S 3$, this is achieved by repeated simulations of the forward and adjoint systems. $G(\tau)$ is computed for a range of $\tau$ and for a range of spanwise wavenumbers $\beta$.

\section{Numerical methods}

To evaluate the evolution operator $\mathscr{A}(\tau)$ and its adjoint $\mathscr{A}^{*}(\tau)$ we follow the 'bifurcation-analysis-for-timesteppers' methodology (Tuckerman \& Barkley 2000) in which a time-dependent nonlinear Navier-Stokes code is modified to perform linear stability analyses and related tasks. Additional details can be found in Barkley et al. (2008).

\subsection{Discretization}

In the present work, the spatial discretization of the Navier-Stokes and related equations is based upon the spectral/ $h p$ element approach. In brief, the computational domain is decomposed into $K$ elements and a polynomial expansion is used in each element. Time discretization is accomplished by time splitting/velocity correction (Karniadakis, Israeli \& Orszag 1991; Guermond \& Shen 2003). Details can also be found in Karniadakis \& Sherwin (2005).

Recall from $\S 2$ that the base flows are two-dimensional and that linear perturbations are Fourier decomposed in the spanwise direction, i.e. (2.14), such that fields $\hat{\boldsymbol{u}}(x, y)$, depending on only two coordinates require computation. Hence throughout this study, we need only a two-dimensional spatial discretization of the step geometry. Following the linear analysis, we carry out DNS for both two-dimensional and threedimensional flows; for three-dimensional solutions, we employ full Fourier modal expansions in the spanwise direction (without the symmetric restriction (2.14)), and the computations are typically performed using a parallelization at the Fourier mode level, with pseudo-spectral evaluation of the nonlinear terms.

Figure 3 shows the domain and spectral-element mesh $M_{1}$ used for the main body of results reported here, two smaller meshes $M_{2}$ and $M_{3}$ used to assess the effect of inflow and outflow lengths $L_{i}$ and $L_{o}$, and details of an additional two meshes $M_{4}$ and $M_{5}$ that were used to check the influence of local mesh refinement at the step edge. The $M_{1}$ domain has an inflow length of $L_{i}=10$ upstream of the step edge and a downstream channel of length $L_{0}=50$. It is worth noting that the inflow length is considerably longer than that required for an eigenvalue stability analysis of the problem. The mesh consists of $K=563$ elements. For a polynomial order of $N=6$, which was applied for most results reported, this corresponds to $563 \times 7^{2} \simeq 28 \times 10^{3}$ nodal points within the domain. A portion of the nodal mesh can be seen in the 

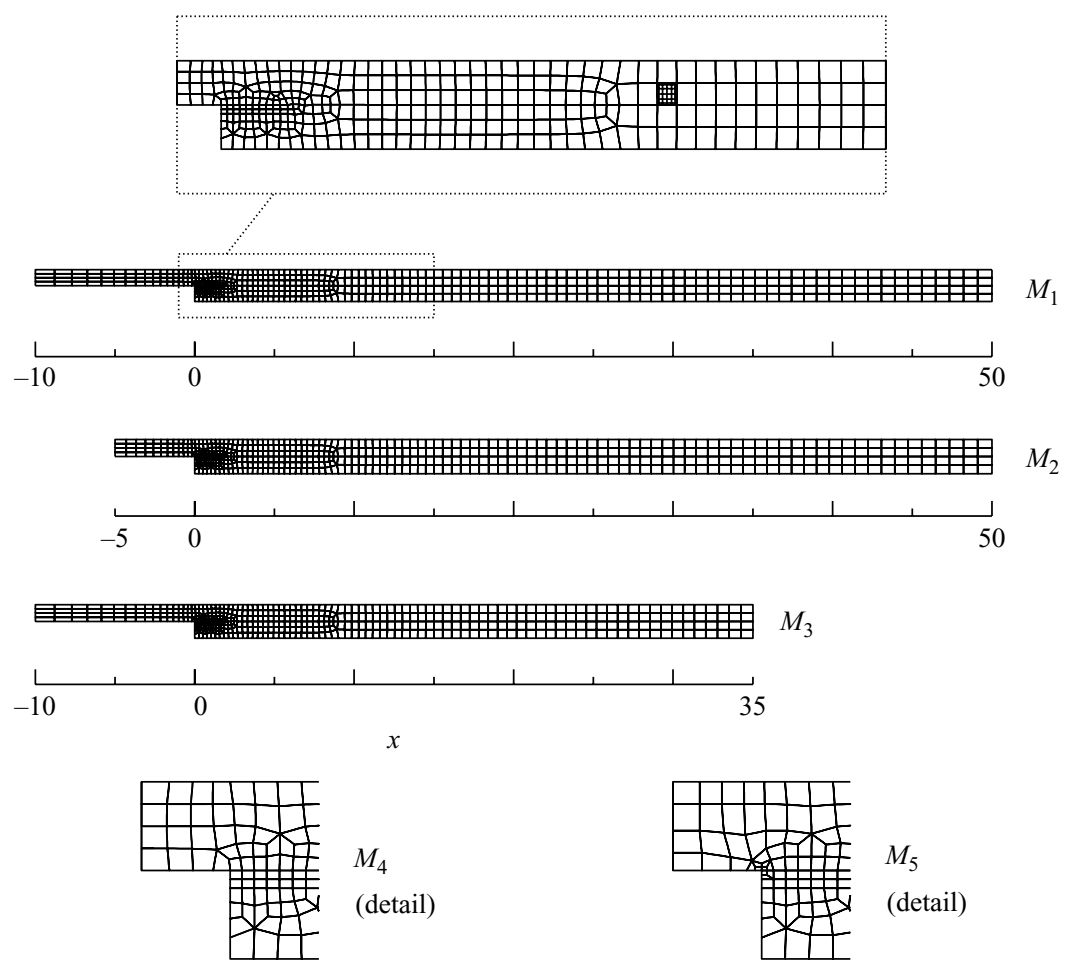

FIGURE 3. Spectral-element meshes for the backward-facing step. The 'production mesh' $M_{1}$ used to compute the main body of results presented here consists of $K=563$ elements; the enlargement shows elements in the vicinity of the step edge and the collocation grid corresponding to polynomial order $N=6$ on a single element. Two smaller meshes $M_{2}$ $(K=543)$ and $M_{3}(K=491)$ have been employed to check the effect of variation in inflow and outflow lengths. Meshes $M_{4}$ and $M_{5}\left(L_{i}=2, L_{o}=35\right)$ were used to examine the effect of local refinement at the step edge.

enlargement at the top of figure 3. In $\S \S 3.3$, and 3.4 we present a convergence study justifying the computational domain parameters.

\subsection{Base and growth computations}

The base flows are steady-state solutions to the Navier-Stokes equations (2.3). We compute these flows simply by integrating the time-dependent Navier-Stokes equations (2.1) to steady state. For the Reynolds-number range of this study, timedependent solutions converge to the steady base flows with reasonable rapidity.

The innermost operation required in computing optimal disturbances is to obtain the actions of the operators $\mathscr{A}(\tau)$ and $\mathscr{A}^{*}(\tau)$. These operators correspond to integrating (2.7) and (2.10) over time $\tau$. Since we are using a scheme which handles the advection terms explicitly in time, up to the level of the advection terms the equations are identical to the incompressible Navier-Stokes equations (2.1) except that the linear advection terms $\mathrm{DN} \boldsymbol{u}^{\prime}$ and $\mathrm{DN}^{*} \boldsymbol{u}^{*}$ appear rather than the nonlinear advection term $\boldsymbol{N}(\boldsymbol{u})=(\boldsymbol{u} \cdot \nabla) \boldsymbol{u}$. The explicit treatment of these terms therefore means that the numerical implementation is easily modified to integrate the forward, adjoint or nonlinear systems. Employing index notation and the summation convention, the 
linearized advection terms for Cartesian coordinates are

$$
\begin{aligned}
\left.(\mathrm{DN} \boldsymbol{u})\right|_{j} & =U_{i} \partial_{i} u_{j}+\left(\partial_{i} U_{j}\right) u_{i}, \\
\left.\left(\mathrm{DN}^{*} \boldsymbol{u}\right)\right|_{j} & =-U_{i} \partial_{i} u_{j}+\left(\partial_{j} U_{i}\right) u_{i} .
\end{aligned}
$$

In our implementation, the steady base flow, $U_{i}$, is read once, and its derivatives are also evaluated and stored for repeated use in the computations of terms (3.1). Since for the present problem $U_{1,2}(x, y, z)=f(x, y)$ and $U_{3}=0$, there are no (non-zero) terms involving $z$-derivatives in (3.1). We recall the combined operator $\mathscr{A}^{*}(\tau) \mathscr{A}(\tau)$ acting on any initial field $\boldsymbol{u}$ is obtained by first integrating the forward system through time $\tau$ followed immediately by integrating the adjoint system through time $\tau$. By (2.13), the initial condition to the adjoint system is precisely the final field from the forward integration.

The outer part of the algorithm to compute optimal disturbances is to find the maximum eigenvalue of $\mathscr{A}^{*}(\tau) \mathscr{A}(\tau)$. This is accomplished using an iterative eigenvalue solver which uses repeated evaluations $\mathscr{A}^{*}(\tau) \mathscr{A}(\tau)$ to determine its maximum eigenvalue. Since the eigenvalues of $\mathscr{A}^{*}(\tau) \mathscr{A}(\tau)$ are real and non-negative, the maximum eigenvalue is dominant (of largest magnitude) and can be found easily by almost any iterative method, including simple power iteration. In practice, we use a Krylov-based method described elsewhere (Tuckerman \& Barkley 2000; Barkley \& Henderson 1996; Barkley et al. 2008). This approach is distinct from that used by Ehrenstein \& Gallaire (2005), and does not involve computing a large number of basis vectors.

There are a few points worthy of further mention. The first is that the only difference between the code used here to compute the optimal perturbation modes and the eigenvalue code used in our previous studies is in the additional evaluation of $\mathscr{A}^{*}(\tau)$ involving the use of $(3.1 b)$. So rather than evaluating only $\mathscr{A}(\tau)$ using $(3.1 a)$, we evaluate $\mathscr{A}^{*}(\tau) \mathscr{A}(\tau)$ using a computation of $(3.1 a)$ and $(3.1 b)$. The second point is that while we are primarily interested in the maximum eigenvalue of $\mathscr{A}^{*}(\tau) \mathscr{A}(\tau)$, for the backwards-facing-step flow that we are considering we shall see that there are two closely related leading eigenvalues and eigenmodes and hence it is useful to compute more of the spectrum of $\mathscr{A}^{*}(\tau) \mathscr{A}(\tau)$ than just the maximum eigenvalue, as would be obtained using the standard power method. Finally, we are interested not only in the optimal initial perturbations, but also the action $\mathscr{A}(\tau)$ on such perturbations. That is, we are interested in the outcome of such perturbations after evolution by time $\tau$. These outcomes may be computed simultaneously in the eigenvalue iterations. In essence, we compute the leading singular value decomposition of $\mathscr{A}(\tau)$, obtaining both the initial modes (right singular vectors) and output modes (left singular vectors); see Barkley et al. (2008).

\subsection{Validation}

We compared the growth predicted by our implementation to values published by Butler \& Farrell (1992), computed using the Orr-Sommerfeld and Squire modes, for plane Poiseuille flow of channel height $2 h$ at $R e=U_{\max } h / v=5000$ (below the asymptotic instability at Reynolds number $R e=5772$ ). The instability modes considered had streamwise wavenumber $\alpha=2 \pi h / L_{x}$ and crossflow wavenumber $\beta=2 \pi h / L_{z}$. In our calculations, sufficient spatial resolution was used to converge the growth values to better than four significant figures. Results presented in table 1 demonstrate good agreement between the two sets of computations.

The tests reported in table 1 give us confidence that the method correctly computes the optimal growth eigenproblem, at least for simple geometries. More generally, we 


\begin{tabular}{ccccc}
\hline$\alpha$ & & & $G(\tau)$ & $G(\tau)$ \\
1.48 & 0.0 & 14.1 & Butler \& Farrell $(1992)$ & Present method \\
3.60 & 7.3 & 5.0 & 45.7 & 45.7 \\
0.93 & 3.7 & 20.0 & 512 & 49.1 \\
& & &
\end{tabular}

TABLE 1. Comparison of growth values computed for instability modes of plane Poiseuille flow at $R e=5000$ by Butler \& Farrell (1992, table III) and by the present method.

$\begin{array}{lccccccc}N & 3 & 4 & 5 & 6 & 7 & 8 & 9 \\ G(60) & 61133 & 61156 & 62989 & 62661 & 62619 & 62626 & 62626 \\ \% \text { difference } & 2.38 & 2.34 & -0.58 & -0.06 & 0.01 & 0.00 & -\end{array}$

TABLE 2. The effect of spectral element polynomial order $N$ on two-dimensional growth at $\tau=60$ for $R e=500$, computed with mesh $M_{1}$.

can also check the energy growth in arbitrary geometries, independent of the adjoint system. To do so we take the computed leading eigenvector of $\mathscr{A}^{*}(\tau) \mathscr{A}(\tau)$, use it as an initial condition for integration of the linearized Navier-Stokes equations over interval $\tau$, and finally compute the ratio of the integrals of the energies in the initial and final conditions. This ratio should be the same as the leading eigenvalue of $\mathscr{A}^{*}(\tau) \mathscr{A}(\tau)$. Testing the energy growth of just the forward integration for $\tau=50$ for the backward-facing step at $R e=450, N=6$ gives:

$$
\frac{E(50)}{E(0)}=\frac{0.0668038}{4.77408 \times 10^{-6}}=13993 \text {. }
$$

For comparison, the equivalent eigenvalue of $\mathscr{A}^{*}(50) \mathscr{A}(50)$ was 13996 , different by $0.02 \%$.

\subsection{Convergence and domain-independence}

Having satisfied ourselves with the veracity of the computational method, we turn to the choices of polynomial order $N$ and domain size (in effect, $L_{i}$ and $L_{o}$ ) used in our study. At the maximum Reynolds number we have used, $R e=500$, the maximum two-dimensional optimal growth occurs near $\tau=60$ and so we consider $R e=500$ and $\tau=50, \tau=60$ in this section. We have restricted the amount of information presented about mesh design to a minimum consistent with building confidence in our choices, but we have investigated over a dozen different designs in undertaking this convergence study. In computing all results presented here we have used second-order time integration with time step $\Delta t=0.005$, and have checked that this is sufficiently small that the outcomes are insensitive to step size.

Table 2 shows the dependence of $G(60)$ on polynomial order $N$ for our production mesh, $M_{1}$. The results are converged to five significant figures at $N=8$, and are only $0.01 \%$ different at $N=7$. We have used $N=7$ to compute the remainder of the results.

Table 3 shows the effect of truncating the domain inflow and outflow lengths, while keeping the inner portion of the mesh (in fact, the extent shown in the inset of figure 3) constant. It can be seen that the effect of either truncating the inflow length $L_{i}$ from 10 to $5\left(M_{2}\right)$ or the outflow length $L_{o}$ from 50 to $35\left(M_{3}\right)$ is small. The 


\begin{tabular}{lrrcc}
\hline Mesh & $L_{i}$ & $L_{o}$ & $G(60)$ & $\%$ difference \\
$M_{1}$ & 10 & 50 & 62619 & - \\
$M_{2}$ & 5 & 50 & 62375 & 0.39 \\
$M_{3}$ & 10 & 35 & 62607 & 0.02
\end{tabular}

TABLE 3. The effect of variation of domain inflow and outflow lengths on two-dimensional growth at $\tau=60, R e=500$, polynomial order $N=7$.

$\begin{array}{lccccc}\text { Mesh } \backslash N & 3 & 4 & 5 & 6 & 7 \\ M_{4} & 52079 & 51439 & 53334 & 53226 & 53147 \\ M_{5} & 52235 & 51286 & 53324 & 53228 & 53146\end{array}$

TABLE 4. The effect local mesh refinement at the step edge on two-dimensional growth at $\tau=50, R e=500$ examined at different spectral element polynomial orders $N$, computed with meshes $M_{4}$ and $M_{5}$.

outflow length has been chosen to allow reliable computations for $\tau>60$, and this is reflected in the fact that the effect on $G(60)$ is minimal.

Finally, it will be observed in later sections that extremely sharp flow features can occur near the step edge. In order to confirm that the results are insensitive to local mesh refinement around the step, once $N$ is sufficiently large, we have used meshes $M_{4}$ and $M_{5}$, both with $L_{i}=2$ and $L_{o}=35$, but where $M_{5}(K=425)$ has an additional level of element refinement around the step compared to $M_{4}(K=419)$, see figure 3. The maximum values of two-dimensional growth for $R e=500, \tau=50$ computed on $M_{4}$ and $M_{5}$ are listed as functions of $N$ in table 4. It can be seen that for $N \geqslant 5$ there is almost no effect of corner refinement. The production mesh $M_{1}$ (also $M_{2}, M_{3}$ ) has the same element structure around the edge as $M_{4}$, and hence is assessed to be adequate for both transient growth analysis and DNS at $N=7$.

\section{Results}

We report on the study of optimal growth, first for two-dimensional, and then for three-dimensional perturbations. For the most part, we find that there is little qualitative difference between the two- and three-dimensional cases, although threedimensional perturbations are energetically favoured, slightly, over two-dimensional perturbations. For the two-dimensional study, we present the optimal growth as a function of both the Reynolds number and the evolution time $\tau$. For the threedimensional study, we fix the Reynolds number and consider the dependence of optimal growth on spanwise wavenumber and the evolution time $\tau$.

\subsection{Base flows}

For completeness, we begin by briefly reviewing the computed base flows. We plot in figure 4 the stagnation points on both the lower and upper walls of the channel as a function of Reynolds number up to 500 . The lower stagnation point marks the limit of the primary recirculation zone behind the step. At $R e \approx 275$, a secondary recirculation zone appears on the upper wall (at $x \approx 8.1$ ), and this gives rise to the appearance of two additional stagnation points. These findings are in quantitative 

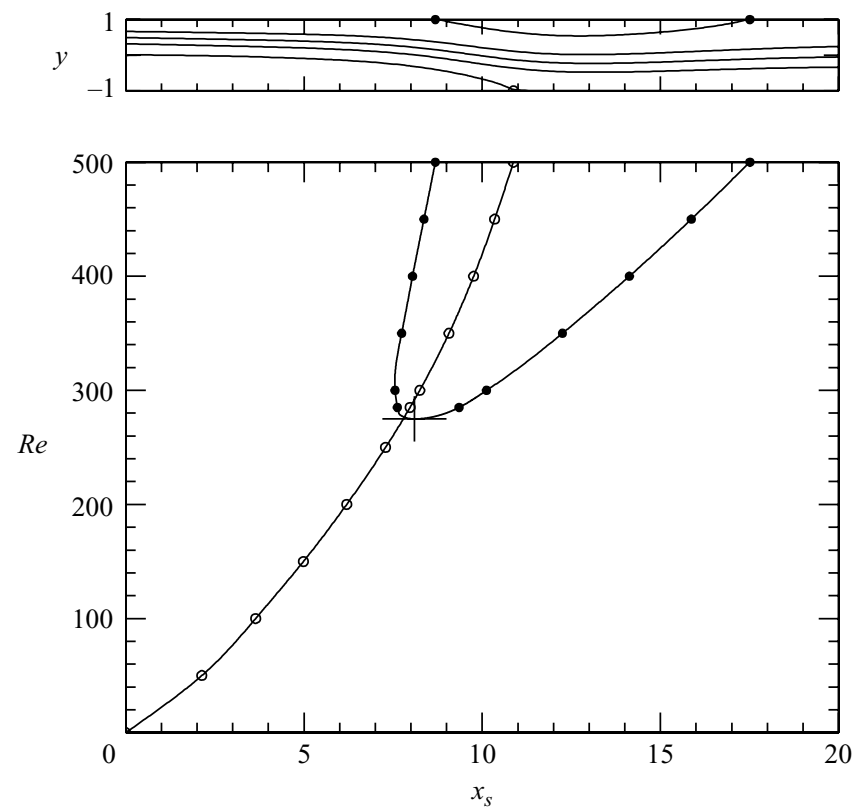

FIGURE 4. Location of stagnation points for the base flows as functions of Reynolds number. Open circles denote stagnation points on the lower channel wall. Solid circles denote the pair of stagnation points on the upper wall associated with the secondary separated zone which forms at $R e \approx 275, x \approx 8.1$ (indicated by a cross). The top panel of the figure shows the relevant portion of the base flow at $R e=500$, illustrated by contours of the streamfunction drawn at $1 / 6$-spaced intervals, with separation points indicated.

agreement with previous computations of flow in this geometry (e.g. Barkley et al. 2002).

\subsection{Two-dimensional optima}

Figure 5 summarizes the results from the two-dimensional optimal growth computations over the full range of times and Reynolds numbers in our study. Figure 5(a) shows optimal envelope curves, $G(\tau)$, for a set of Reynolds numbers in equal increments from $R e=50$ to 500. Note that at Reynolds numbers less than approximately 50, there is no growth for any time interval. Peak growth, and the time (hence, streamwise location) at which it occurs, increases monotonically with Reynolds number. Figure $5(b)$ presents $G$ as a contour plot in the $(\tau, R e)$-plane. Everywhere outside of the darkest grey region, $G$ is larger than unity, corresponding to transient energy growth.

Two important initial observations on these data are the following. First, at $R e=$ 500 , the maximum Reynolds number considered in this study and well below the value $R e \simeq 750$ for linear asymptotic instability of the flow (Barkley et al. 2002), there exist perturbations which grow in energy by a factor of more than $60 \times 10^{3}$. Secondly, the critical Reynolds number, $R e_{c}$, demarcating where $G$ first exceeds unity, is comparatively small. The value $R e_{c}$ is given by the Reynolds number such that $\partial G /\left.\partial \tau\right|_{\tau=0}=0$. From detailed computation in the vicinity of $R e_{c}$ we have determined $R e_{c}=57.7$, to within $1 \%$ accuracy. $R e_{c}$ is indicated in figure $5(b)$ and is the Reynolds number at which the $G=1$ contour meets the Reynolds-number axis. In figure $5(a)$, we can see that the optimal curve $G(\tau)$ at $R e=50<R e_{c}$ is monotonically decreasing with $\tau$, while for $R e=100>R e_{c}$ the optimal curve has positive slope at $\tau=0$ 

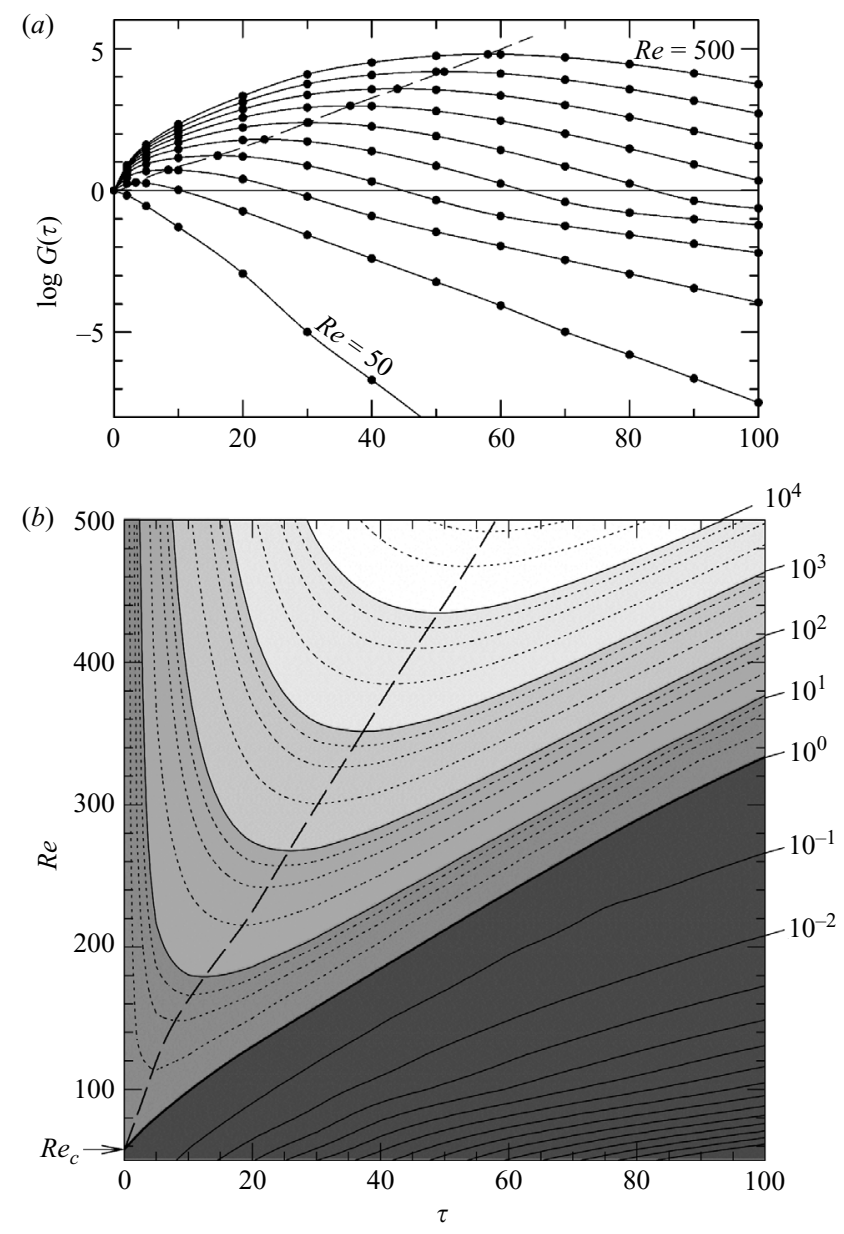

FIGURE 5. (a) Two-dimensional optima as functions of $\tau$ for Reynolds numbers from 50 to 500 in steps of 50. The maximum growth at $R e=500$ is $G(58.0)=63.1 \times 10^{3}$. (b) Contour plot for two-dimensional optimal growth $G$ as a function of $\tau$ and $R e$. Lighter grey regions correspond to positive growth (i.e. $G>1$ ). Solid contour lines are drawn at decade intervals, as indicated. Decay (i.e. $G<1$ ) is indicated with the darkest grey region. The critical Reynolds number for the onset of transient growth is $\operatorname{Re}_{c}=57.7$. In both $(a)$ and $(b)$, a dashed-line curve shows the locus of global maximum growth as a function of $\tau$.

and there is a range of $\tau$ for which $G>1$, i.e. the energy of an optimal disturbance increases, rather than decreases, from its initial value.

For reference we show in figure 6 the optimum envelope $G(\tau)$ at $R e=500$, on a linear scale, together with three transient responses. The three transients follow from those initial conditions which produce optimal energy growth at $\tau=20, \tau=60$ and $\tau=100$. These curves necessarily meet the optimum envelope at the corresponding times, as shown. Such plots are typically presented in optimal growth studies. Figure 6 emphasizes the idea that the optimal curves (such as are shown in figure $5 a$ ), represent envelopes of individual transient responses. However, as a practical matter, many of the individual transients which follow from optimal initial conditions correspond qualitatively and approximately quantitatively to the envelope. In particular, for $R e=500$, transient responses starting from the optimal perturbation corresponding 


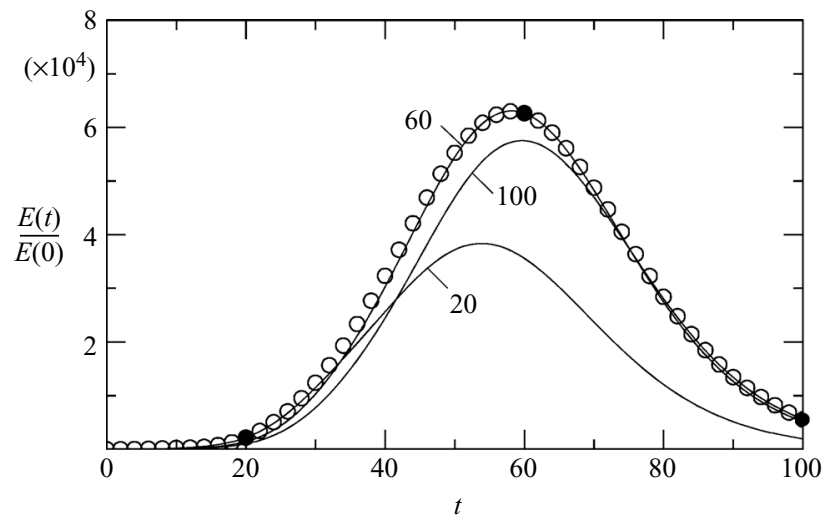

FiguRE 6. The envelope of two-dimensional optima (circles) at $R e=500$ together with curves of linear energy evolution starting from three optimal intial conditions for specific values of $\tau: 20,60$ and 100. Solid circles mark the points at which the curves of linear growth osculate the envelope.

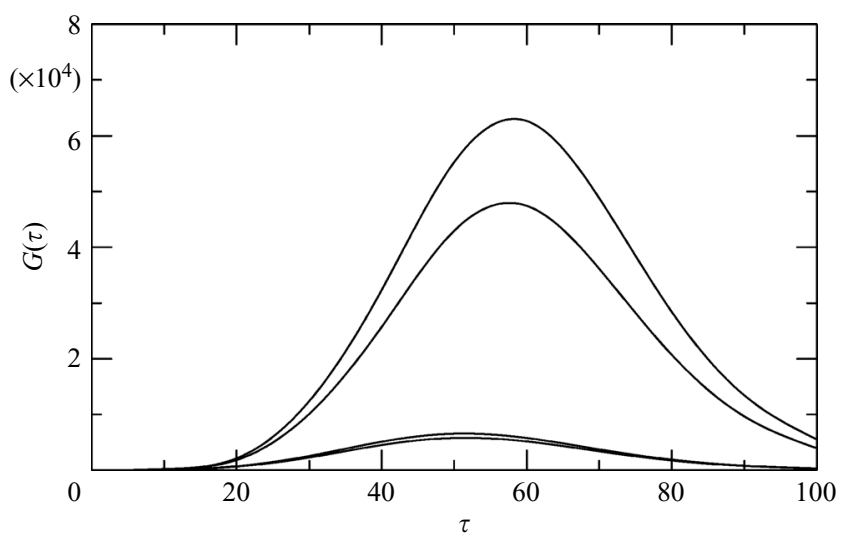

FIGURE 7. The growth envelopes of the optimal and three leading sub-optimal two-dimensional disturbances at $R e=500$.

to $\tau \approx 60$ are nearly indistiguishable from the optimal envelope $G(\tau)$ shown in figure 6. We note, however, that such behaviour is not ubiquitous: for flows where more than one instability mechanism is present, individual responses may be quite different to the envelope (see e.g. figure 7, Corbett \& Bottaro 2000).

\subsubsection{Sub-optimal growth}

As noted in $\S 3$, we may compute not only the leading eigenvalue of $\mathscr{A}^{*}(\tau) \mathscr{A}(\tau)$, corresponding to $G(\tau)$, but also sub-optimal eigenvalues. In the present case, it is worth considering the first sub-optimal eigenvalues because they are close in value to the leading ones (for reasons that will become clear from results and discussion in $\S \S 4.2 .2$ and 5.1). In figure 7, we show both the optimal and first three sub-optimal growth envelopes at $R e=500$ and in table 5 we show four leading eigenvalues of $\mathscr{A}^{*}(60) \mathscr{A}(60)$. We see that there is a pair of eigenvalues well separated in magnitude from the remaining ones. While we have not computed the leading four eigenvalues everywhere in our parameter study (as presented in figure 5), whenever we examined the ranking of eigenvalues in detail we found this structured ordering. 


\begin{tabular}{cccc}
$\lambda_{1}$ & $\lambda_{2}$ & $\lambda_{3}$ & $\lambda_{4}$ \\
$62.7 \times 10^{3}$ & $47.4 \times 10^{3}$ & $5.8 \times 10^{3}$ & $5.2 \times 10^{3}$ \\
TABLE 5. The four largest eigenvalues of $\mathscr{A}^{*} \mathscr{A}$ for two-dimensional perturbations at \\
\multicolumn{4}{c}{$\operatorname{Re}=500, \tau=60}$. \\
\hline
\end{tabular}

(a)

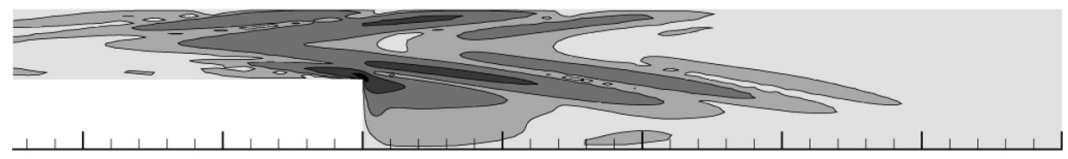

(b)

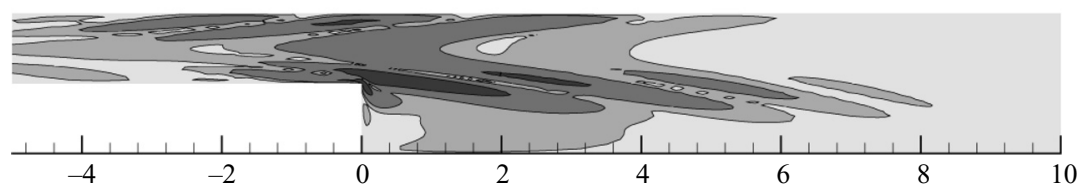

(c)

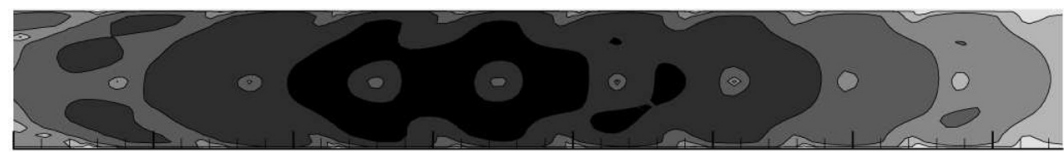

(d)

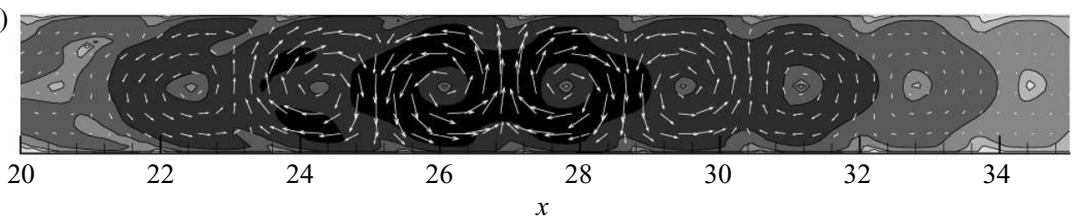

FIGURE 8. Contours of the logarithm of energy in the two-dimensional $(a)$ optimal disturbance initial condition at $R e=500 ;(b)$ in the leading sub-optimal disturbance, and $(c, d)$ in the corresponding linear growth outcomes at $\tau=58.0 ;(d)$ has the velocity vector field overlaid. Contours are drawn at decade intervals.

\subsubsection{Perturbation fields}

We now consider the perturbation fields associated with optimal growth. In figure $8(a)$, we plot contours of energy in the global optimum two-dimensional disturbance for $R e=500$, for which $\tau=58.0$. We also plot (figure $8 b$ ) the eigenmode corresponding to the second eigenvalue. Unsurprisingly, the energies of these eigenfunctions are concentrated near the step edge - note that the energy contours in figure 8 are drawn to a logarithmic scale, and that there are very sharp peaks right at the edge. In figure $8(c, d)$, we plot contours of energy in the perturbation solutions that linearly evolve from these two disturbances, at $t=58.0$ which is when two-dimensional global maximum growth occurs (this outcome is figure $8 c$ ). The flow structures that give rise to these energy contours are a series of counter-rotating spanwise rollers; energy minima near the centreline of the channel correspond to the centres of the rollers. The production of energetic spanwise rollers through tilting of initially highly strained and backward-leaning structures that arise near the walls is very similar to what is observed for two-dimensional optimal growth in plane Couette and Poiseuille flows (Farrell 1988; Schmid \& Henningson 2001).

Note that the locations of maximum energy in the first sub-optimal disturbance, figure $8(b)$, interleave those in figure $8(a)$. As these features evolve into rollers, we see in 


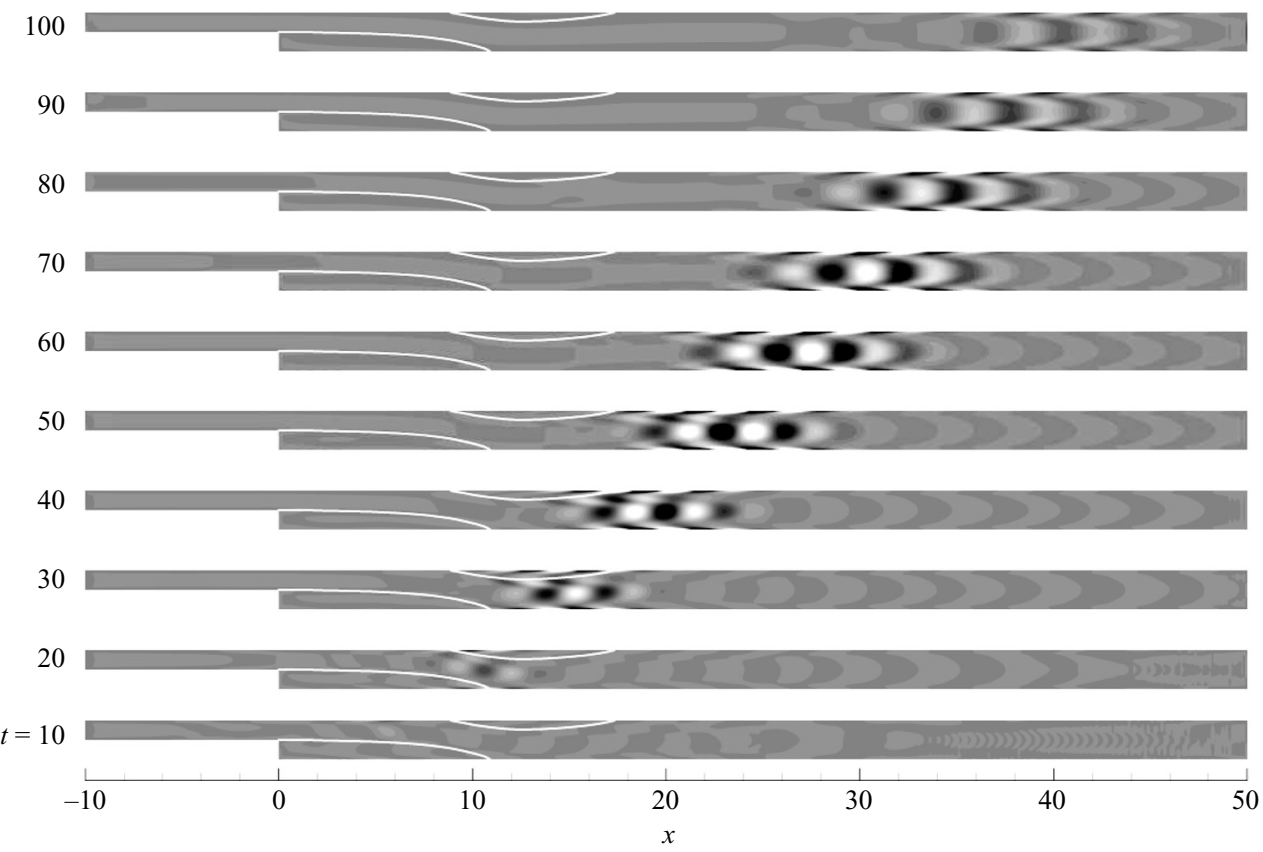

FIGURE 9. Sequence of linear perturbation vorticity contours developed from the two-dimensional global optimum disturbance initial condition for $R e=500$ (maximum energy growth occurs for $t=\tau=58.0$ ). Separation streamlines of the base flow are also shown. The characteristic space-time dynamics of a local convective instability is clearly evident.

figure $8(c, d)$ that the energy maxima still have this property. The operators $\mathscr{A}^{*}(\tau) \mathscr{A}(\tau)$ and $\mathscr{A}(\tau) \mathscr{A}^{*}(\tau)$ are both self-adjoint and hence the eigenfunctions for each operator, corresponding to distinct eigenvalues, are orthogonal. The pair of disturbance initial conditions in figures $8(a)$ and $8(b)$ are eigenfunctions of $\mathscr{A}^{*}(\tau) \mathscr{A}(\tau)$ and hence are orthogonal to one another. Likewise the output disturbances in figures $8(c)$ and $8(d)$ are eigenfunctions of $\mathscr{A}(\tau) \mathscr{A}^{*}(\tau)$ and hence are also orthogonal to one another.

Figure 9 shows a sequence of perturbation vorticity contours that evolve from the two-dimensional global optimum disturbance initial condition for $R e=500, \tau=58.0$ (i.e. figure $8 a$ ). The characteristic dynamics of a locally convectively unstable flow, as illustrated in figure $1(c)$, is clearly evident in this plot. The evolved roller structures are seen clearly for times between 50 and 70 , and for $x$ in the range 25 to 30 . At early times, $t \lesssim 40$, the disturbance traverses past the two separation bubbles and it appears that there is some interaction (e.g. at $t=30,40$ where perturbation vorticity can be seen around the upper separation streamline). As the roller structures decay at large times they are distorted by the mean strain field into approximately parabolic shapes in a process that continues the tilting of the initial disturbances.

Figure 10 (based on the same simulation data as figure 9) shows the centroidal locations of energy in a perturbation that grows from the two-dimensional global optimum disturbance at $R e=500$. At $t=58.0$, the centroidal location is $x_{c}=26.4$ (cf. figure $8 \mathrm{c}$ ); subsequently, the location of the centroid moves downstream at approximately $U_{\infty} / 3$, which is the average speed of Poiseuille flow in the expanded channel. 


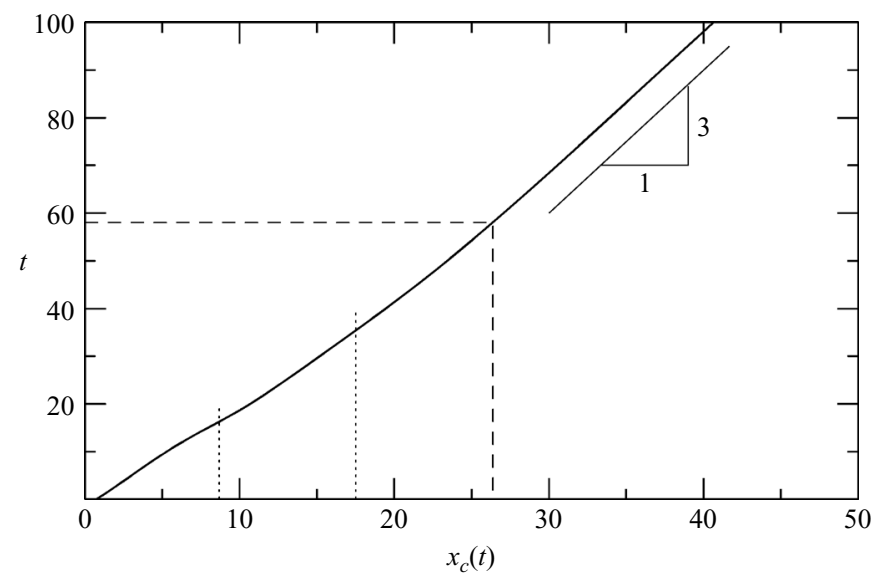

FIGURE 10. The location $x_{c}$ of the centroid of the perturbation energy evolving from the two-dimensional global optimal disturbance as a function of time for $R e=500$. Dotted lines indicate the extent of the upper separation bubble, the dashed lines indicate centroidal location and time increment for peak growth, while the line of slope $3: 1$ indicates the asymptotic average dimensionless flow speed in the downstream channel.

\subsubsection{Reynolds-number dependence}

Here we address the Reynolds-number dependence of the maximum obtainable growth and the associated disturbances. For a given $R e$, we define $G_{\max }=\max _{\tau} G(\tau)=$ $G\left(\tau_{\max }\right)$ as the maximum possible energy growth. These maxima are indicated by the dashed lines in figure 5. In figure 11 $(a, b)$, we plot the dependence of $\tau_{\max }$ and $G_{\max }$ on $R e$. Figure 11(b) shows that over the range of parameters in our study, $G_{\max }$ grows exponentially with $R e$. From the slope of the logarithmic curve, we find that $G_{\max }$ increases by a factor of approximately 15 for each $R e$ increment of 100 .

At each value of $R e$ there is an initial condition and disturbance corresponding to $G_{\max }$. For each such disturbance, we find the location of the energy centroid at the optimal time: $x_{c}\left(\tau_{\max }\right)$. (The dashed line in figure 10 illustrates this at $R e=500$ where $x_{c}\left(\tau_{\max }=58.0\right)=26.4$.) Figure $11(c)$ shows how $x_{c}\left(\tau_{\max }\right)$ varies with $R e$. For Re below $R e \approx 150$, the centroid $x_{c}\left(\tau_{\max }\right)$ lies upstream of the primary stagnation point. For larger $R e$, the centroid lies downstream of not only the primary stagnation point, but also the secondary stagnation points which form at $R e \approx 275$. For large $R e$, we observe $\partial x_{c} / \partial R e \approx 0.06$. This can largely be accounted for by the variation of the optimal time $\tau_{\max }$ with $R e$ : from figure $11(a), \partial \tau_{\max } / \partial R e \approx 0.14$. Taking into consideration that the centroid travels at a speed of approximately $\partial x_{c} / \partial \tau_{\max }=U_{\infty} / 3$ (figure 10) in the downstream channel, we obtain $\partial x_{c} / \partial R e \approx 1 / 3 \times \partial \tau_{\max } / \partial R e \approx 0.05$.

\subsubsection{Weakly nonlinear analysis}

We now investigate weakly nonlinear effects on the optimal linear growth via two-dimensional DNS. Figure 12 presents the perturbation energy evolution for twodimensional DNS at $R e=500$ where the initial condition was the steady base flow seeded with the two-dimensional global optimum disturbance initial condition at three different energy levels. The relative amount of perturbation is quantified by the ratio of the energy in the perturbation to the energy in the base flow within $\Omega$ (domain $M_{1}$ in §3.1). Note that the growth $G(t)$, also a ratio, is the perturbation energy normalized by the intial perturbation energy. For a very small amount of perturbation (ratio $1 \times 10^{-9}$ ) the nonlinear evolution is almost indistinguishable from the linear result. 

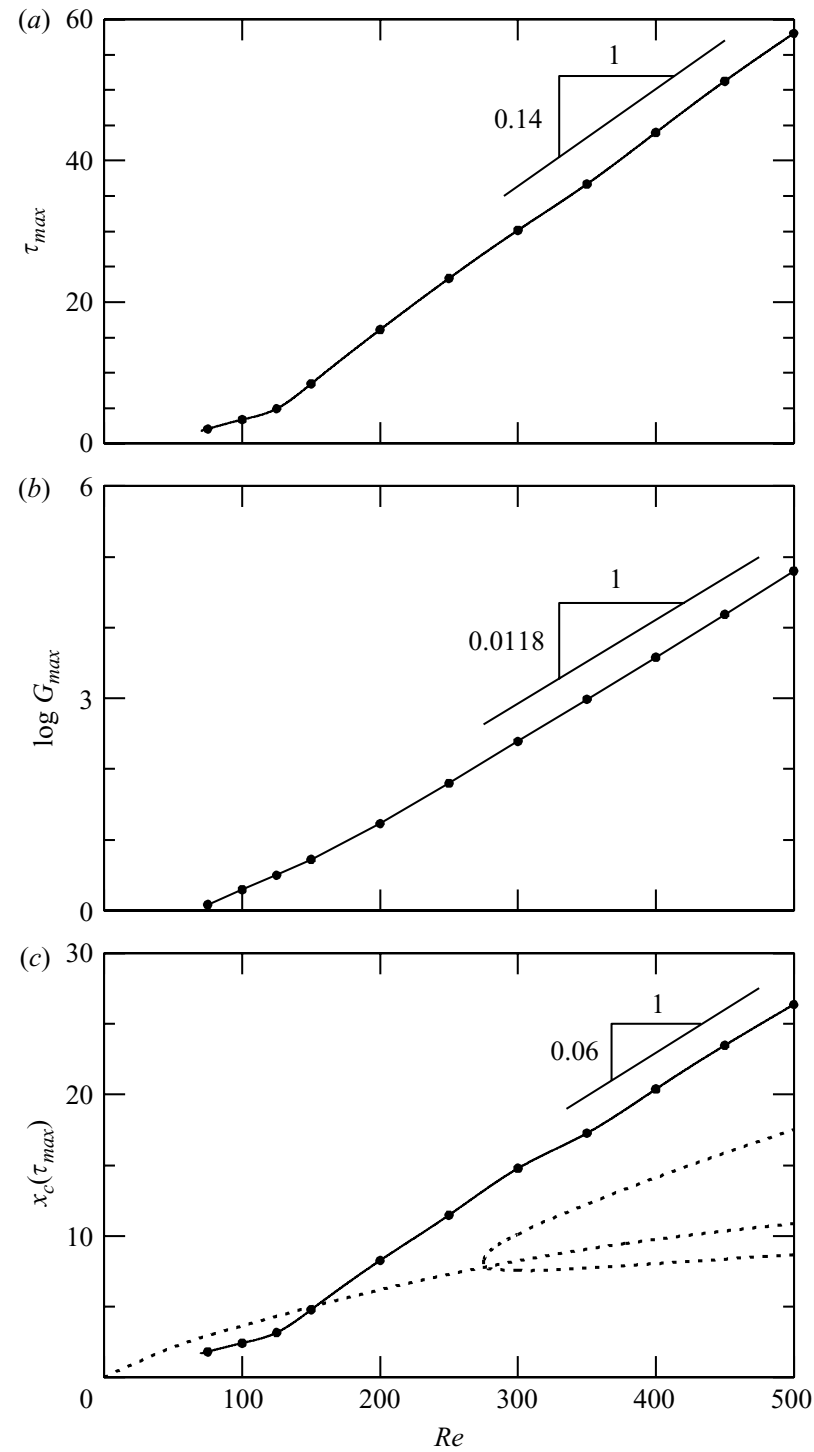

FIGURE 11. Re-dependence of various quantities for two-dimensional flow. $(a)$ The time $\tau_{\max }$ of the energy maximum. (b) The maximum energy growth $G\left(\tau_{\max }\right)$. The asymptotic slope of $\partial \log G\left(\tau_{\max }\right) / \partial R e=0.0118$ means that the value of $G\left(\tau_{\max }\right)$ grows by 15.1 when $R e$ is incremented by 100. (c) The centroid location $x_{c}$, with stagnation points of the base flow indicated as dotted curves.

As the relative amount of perturbation increases, the time for peak energy growth decreases, and the peak growth (but not the peak perturbation) also decreases. These results show that as nonlinearity comes into play, it has a stabilizing effect on the growth of perturbations.

\subsection{Three-dimensional optima}

Now the scope of possible perturbations is broadened to allow spanwise variation (equations (2.14)), with the additional parameter $\beta=L_{z} / 2 \pi$, the spanwise wavenumber. We choose to fix the Reynolds number at $R e=500$. Figure 13 shows 


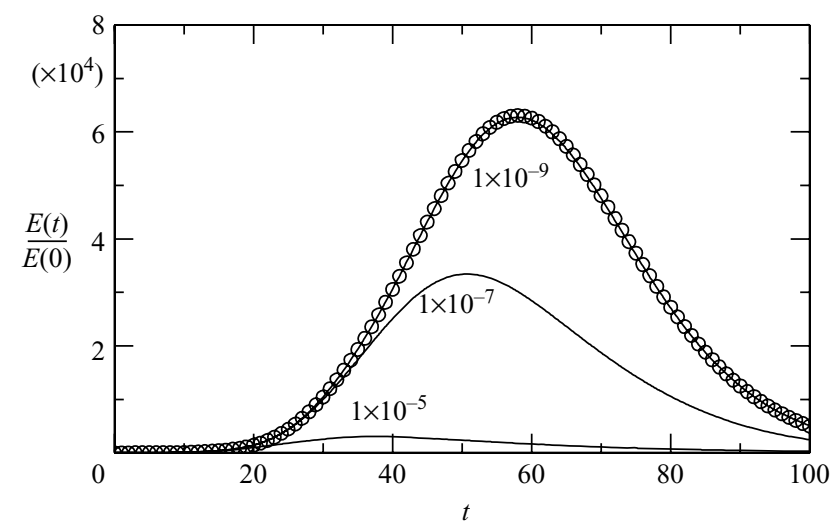

FIGURE 12. Nonlinear effect on energy growth in two-dimensional flow at $R e=500$. Open circles show linear energy growth for the global optimum disturbance. Solid lines are obtained from DNS where the labels indicate the ratio of the integral over the domain area of the perturbation energy to the integral of the energy in the base flow.

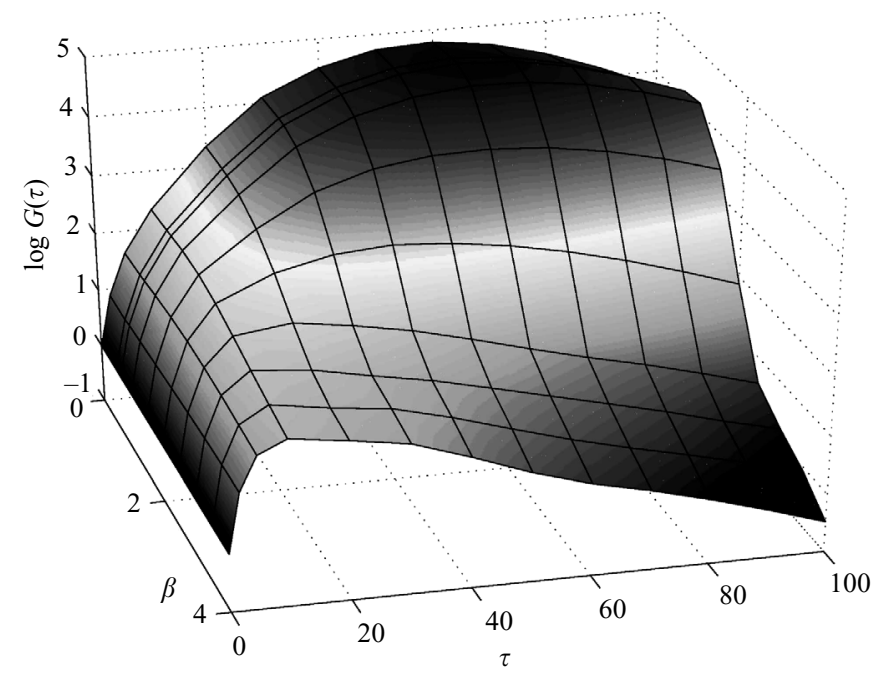

FIGURE 13. Surface plot of $R e=500$ three-dimensional optimal growth $G(\tau, \beta)$ where $\beta$ is spanwise wavenumber. Maximum growth $G(61.9,0.645)=78.8 \times 10^{3}$.

the optimal growth as a function of parameters $\tau$ and $\beta$ : the $G(\tau, \beta)$ surface. From these data we extract $G_{\max }$, now the maximum value of $G(\tau)$ over all $\tau$ at a given wavenumber. In figure 14, we plot the resulting maxima as a function of wavenumber. These maxima initially increase as a function of $\beta$, reaching a global maximum at wavenumber $\beta=0.645$. Above $\beta \approx 1$, the maximum growth falls significantly, by approximately three orders of magnitude from $\beta=1$ to $\beta=4$. In figure 13 , it can be seen that this fall is principally owing to the drop in optimal growth for $\tau \gtrsim 10$ at large $\beta$, with the result that the maximum growth occurs for much earlier durations (and so, will be associated with smaller values of $x$ ).

In figure 15 , we plot $G(\tau, \beta=0.645)$, the optimal growth envelope at wavenumber $\beta=0.645$. For comparison, the two-dimensional envelope $G(\tau, \beta=0)$ is also shown. The shapes of the two envelopes are very similar, especially for $\tau \leqslant 50$, although 


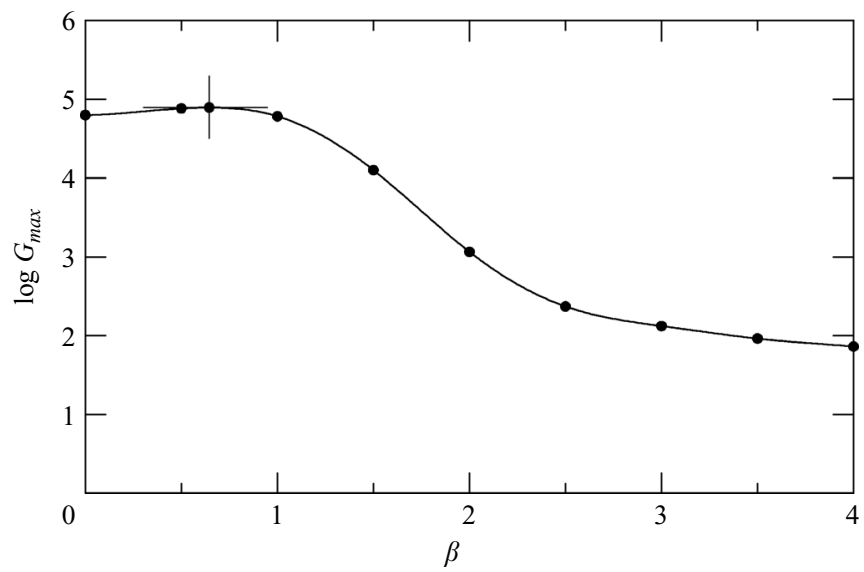

FIGURE 14. Maximum three-dimensional energy growth at $R e=500$ as a function of spanwise wavenumber $\beta$. The wavenumber of the most-amplified disturbance is $\beta=0.645$.

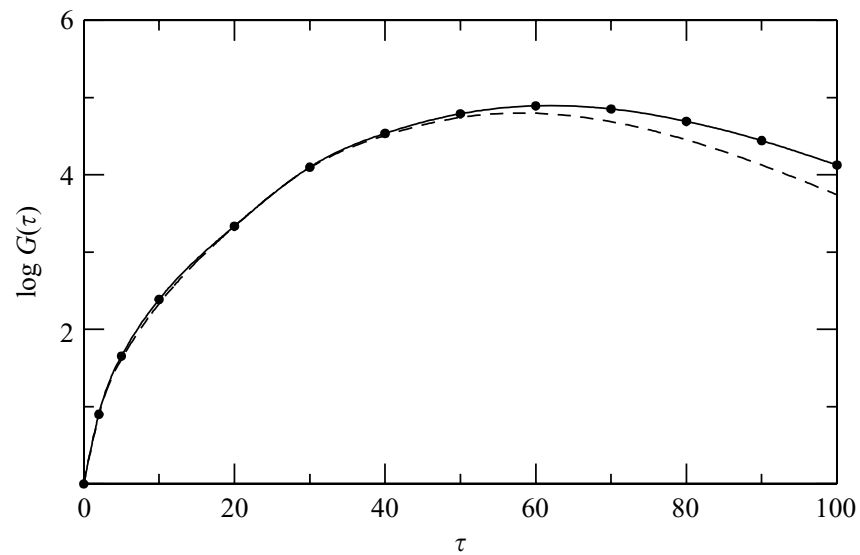

FIGURE 15. Three-dimensional growth optima as functions of $\tau$ at $R e=500$ for the most-amplified spanwise wavenumber, $\beta=0.645$. The dashed line indicates the corresponding two-dimensional result $(\beta=0)$.

the three-dimensional envelope peaks higher and at larger $\tau$ than does the twodimensional envelope.

In summary, the global optimal energy growth at $R e=500$, over all perturbations, is $G\left(\tau_{\max }=61.9, \beta=0.645\right)=78.8 \times 10^{3}$. For comparison, the strictly two-dimensional optimum is $G(58.0,0)=63.1 \times 10^{3}$.

\subsubsection{Three-dimensional perturbation fields}

Figure 16 shows contours of energy in the two leading disturbance initial conditions and the maximum perturbations that grow from them, for $R e=500, \beta=0.645$; this figure is the three-dimensional equivalent of figure 8. Again the optimal and leading sub-optimal initial disturbances are concentrated around the step edge, and the maximum-energy outcomes are a pair of wavy structures that lie in streamwise quadrature to each other. The centroidal location of peak energy for $R e=500$, $\beta=0.645, \tau=61.9$ is $x_{c}=26.6$, similar to the result for the two-dimensional optimum, which at $\tau=58.0$ has $x_{c}=26.4$. 
(a)

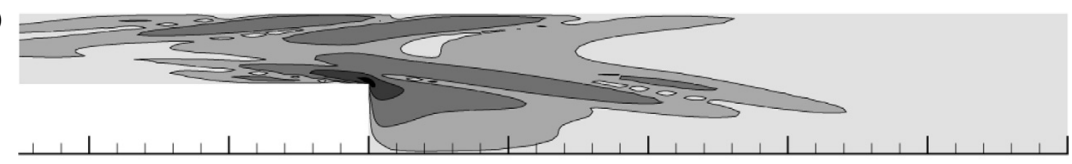

(b)

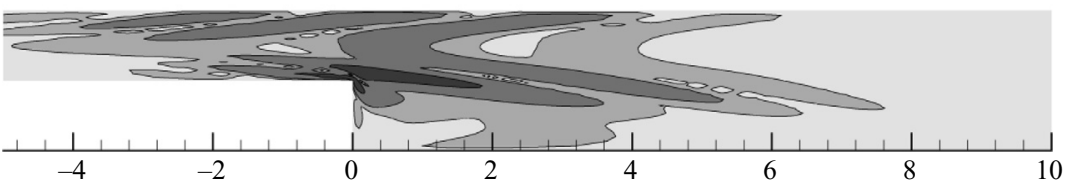

(c)

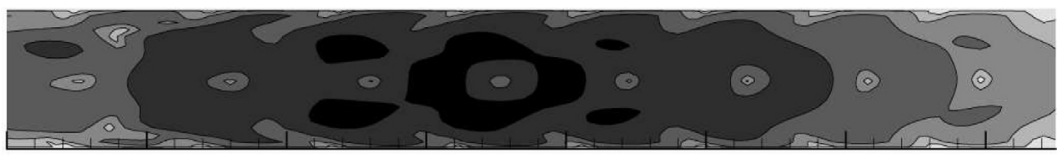

(d)

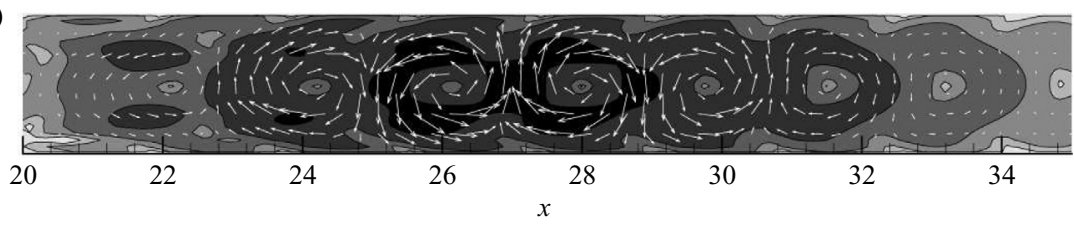

FIGURE 16. Contours of the logarithm of energy in the $\beta=0.645$, three-dimensional ( $a$ ) optimal disturbance at $R e=500$; (b) leading sub-optimal disturbance, and $(c, d)$ of the corresponding linear growth outcomes at $\tau=61.9 ;(d)$ has the $(x, y)$ components of the velocity vector field overlaid. Contours are drawn at decade intervals. Note the general similarity to the two-dimensional results of figure 8.

Figure 17 shows profiles of the vertical $(y)$ velocity component in the two leading three-dimensional transient growth disturbances and their outcomes, extracted along the line $y=0, z=0$. In all cases, the profiles are normalized to have absolute maximum value of unity. The resemblance of the profiles of figure $17(c, d)$ to the 'generic' local convective instability schematics of figure $1(c)$ is readily apparent. The extremely sharp fluctuations in the optimal disturbance at the step edge, previously alluded to in $\$ 3$, can be seen in figure $17(a)$. The average streamwise wavelength of the fluctuations in figure $17(c, d)$, estimated by zero-crossing analysis, is $L_{x} \approx 3.73$, and this is at least qualitatively the same as the streamwise length scale of the initial disturbances seen in figure $17(a, b)$. In addition, we observe that the wavelength is approximately two channel heights, or what would be expected for a pair of circular counter-rotating vortices that fill the channel, much as can be seen in figure $16(d)$.

\subsubsection{Three-dimensional DNS}

Figure 18 shows the evolution of energies in 16 Fourier modes from a threedimensional DNS at $R e=500$. The wavenumbers $\beta_{k}$ in the simulation are multiples of $\beta_{k=1}=0.645$, the wavenumber for optimal three-dimensional growth. The initial condition is the two-dimensional base flow (mode number $k=0$ ), seeded with the global optimum disturbance (mode number $k=1$ ) at relative energy level $1 \times 10^{-9}$. All other mode numbers are initialized at zero. From related two-dimensional results (figure 12), we expect that for this low seeding level the energy evolution in mode number $k=1$ would be little different to what would be obtained in a linear evolution, and this is confirmed by the growth of energy for $k=1$ (approximately $73 \times 10^{3}$ ) and the time for maximum growth $(t \approx 61)$. Energy is transferred to modes $k>1$ via nonlinear interactions. 
(a)

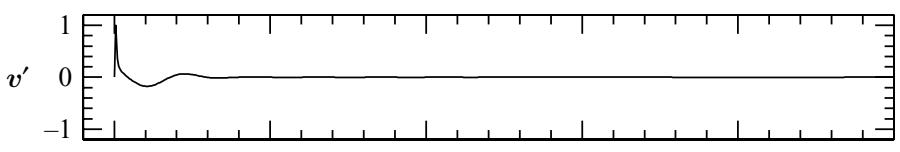

(b)

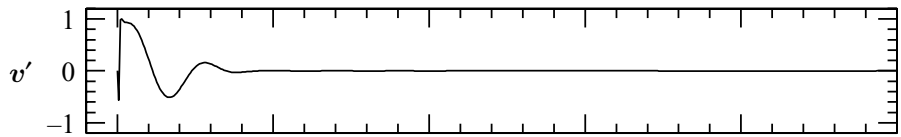

(c)

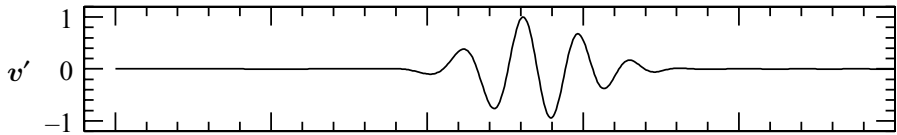

(d)

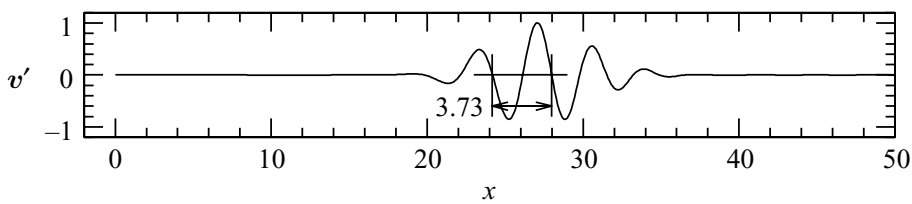

FIGURE 17. Profiles of vertical perturbation velocity component along the line $y=0, z=0$ for $\operatorname{Re}=500, \beta=0.645, \tau=61.9$, corresponding to the cases shown in figure 16. (a) Maximal growth initial condition; $(b)$ leading sub-optimal initial condition. $(c, d)$ show the outcomes of linear growth for these two modes at $\tau=61.9$. Velocities are arbitrarily scaled to have a maximum value of unity. Streamwise length scale of oscillation in $(c, d): L_{x} \approx 3.73$.

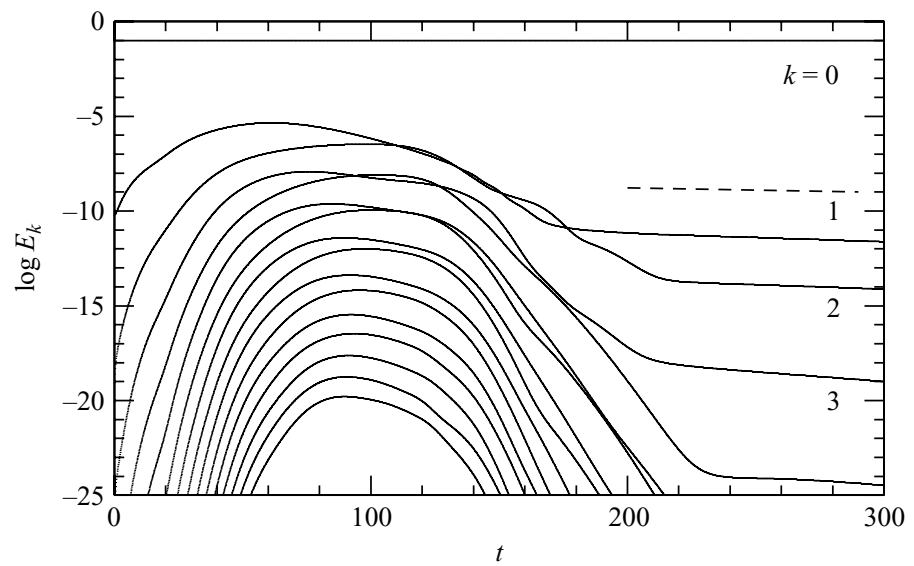

FiguRE 18. Time series of energies in spanwise Fourier modes, $\beta_{k}$ for DNS at $R e=500$, $\beta_{k=1}=0.645$. The relative energy in the initial perturbation, mode $k=1$, is lower than that in the two-dimensional flow $(k=0)$ by a factor of $1 \times 10^{-9}$. Peak energy amplification for $k=1$ occurs at $t=61$, almost the same as predicted for linear transient growth $(t=61.9)$. The dashed line indicates the computed decay rate of the leading asymptotic instability eigenmode.

After approximately 200 time units (by which stage, energy in the leading threedimensional mode has declined below its initial value) the asymptotic decay of energy in $k=1$, and thereafter, higher modes, becomes exponential. Computing the asymptotic linear decay rate for $R e=500, \beta=0.645$ (as in Barkley et al. 2002) we find the leading eigenvalue to be $\lambda=-5.73 \times 10^{-3}$. This decay rate is drawn as a dashed line in figure 18: it matches almost exactly the observed asymptotic decay rate, confirming the expectation that at large times, after transient growth passes, the 
(a)

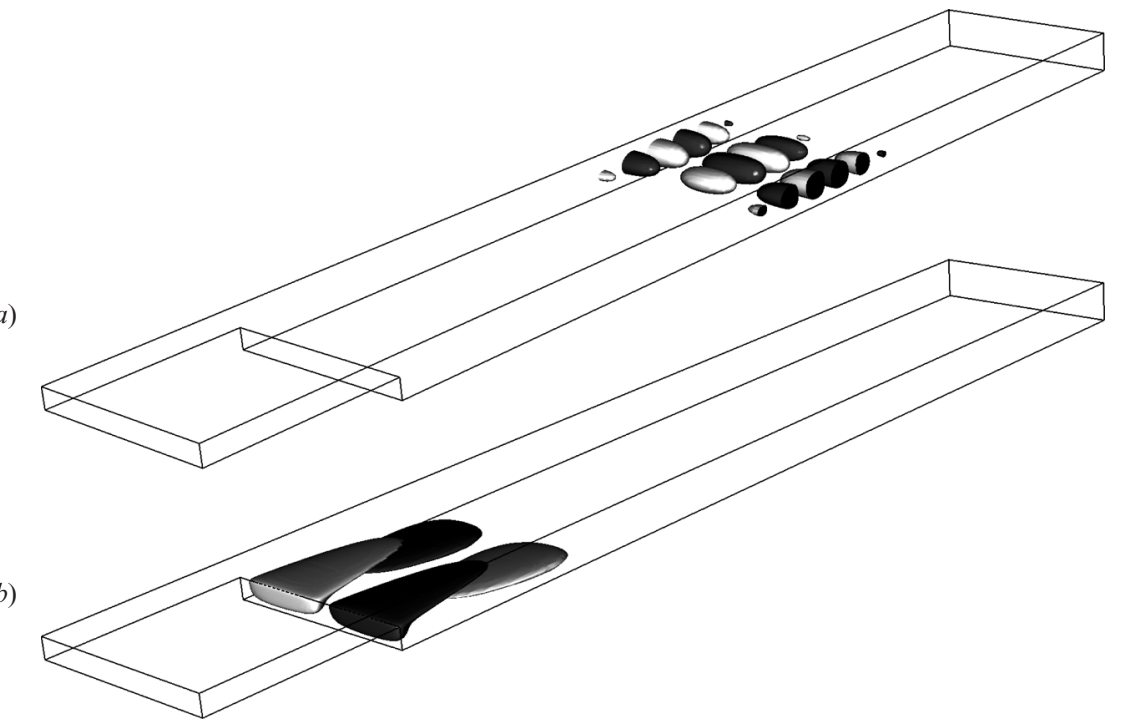

FIGURE 19. Isosurfaces of perturbation velocity components from three-dimensional DNS seeded with the optimal growth initial condition at relative energy level $1 \times 10^{-9}$ (figure 18). (a) Positive/negative isosurfaces of the pertubation vertical velocity component at $t=61$, corresponding to maximum perturbation energy growth. (b) Isosurfaces of the spanwise velocity component at $t=300$ (and at much lower values than in $(a)$ ), when the remaining perturbation is dominated by decay of the leading asymptotic instability mode.

response is dominated by the leading asymptotic mode predicted using the methods of traditional stability analysis.

Figure 19 shows velocity component isosurfaces for times $t=61$ and $t=300$, obtained from the same simulation as used to generate data for figure 18. Figure 19(a) shows positive/negative isosurfaces of vertical perturbation velocity component at $t=61$, when perturbation energy growth is greatest. These isosurfaces show the perturbation energy to be contained in a wave packet well downstream of the step, in the vicinity of $x=25$, as expected from the optimal growth analysis. For $t=300$, the isosurfaces of spanwise velocity shown in figure $19(b)$ suggest that the remaining three-dimensional energy is confined instead to the region of the two separation bubbles, as expected from the asymptotic result quoted above and from the work of Barkley et al. (2002).

\section{Discussion}

Our results confirm that the flow past a backward-facing step of expansion ratio two can exhibit large transient growth at Reynolds numbers well below that for asymptotic instability, and that this growth can be predicted within the framework of linear optimal perturbations, so providing theoretical underpinning for the largely phenomenological study of local convective instability in previous investigations of backward-facing step flows (e.g. Kaiktsis, Karniadakis \& Orszag 1991, 1996; Gresho et al. 1993).

\subsection{Observations on optimal disturbances and mechanisms}

Optimal disturbance initial conditions for the backward-facing step flow have energy sharply concentrated around the step edge, and like the two-dimensional optimal 
perturbations for plane Poiseuille and Couette flows (Farrell 1988; Butler \& Farrell 1992), have a structure that consists of highly strained counter-rotating rollers whose inclination opposes the mean shear. While three-dimensional disturbances are moderately favoured, their crossflow wavelengths are large, of the order of ten step-heights, and their structure is broadly similar to those for the two-dimensional restriction (cf. figures 8 and 16). This is unlike the situation that holds for plane Couette and Poiseuille flows (albeit at larger $R e$ ), where three-dimensional optimal disturbances consisting of streamwise-aligned rollers have much greater energy amplification than do two-dimensional optimal disturbances. For example in plane Poiseuille flow at $R e=5000$ (i.e. a decade higher than the maximum used in the present work) Butler \& Farrell (1992, table III) cite $G_{\max }=45.7$ for the optimal two-dimensional perturbation and $G_{\max }=4897$ for the optimal three-dimensional perturbation. In the backward-facing-step flow, optimal disturbances predominantly gain energy through tilting by the mean shear, i.e. via the inviscid Orr mechanism (Orr 1907; Lindzen 1988), the same mechanism that provides transient energy growth in two-dimensional Couette and Poiseuille flows. In addition, there may be energy growth via cooperative interaction with Kelvin-Helmholtz instabilities in the two separated shear layers (see figure 9 and related text). We note, however, that a direct comparison to Couette and Poiseuille flows may be non-trivial since for these the base flow is independent of Reynolds number, unlike that for the backward-facing step.

The structure of the eigenvalues in figure 7 and the modes in figures 8 and 16 can be thought of as arising from a splitting of the spectrum of $\mathscr{A}^{*}(\tau) \mathscr{A}(\tau)$ owing to broken translational symmetry in the streamwise direction. Had the flow been symmetric in the streamwise direction, then the modes would necessarily come in pairs with trigonometric dependence, i.e. sine and cosine, with equal growth rates (degenerate eigenvalues). Translational symmetry is broken by the step, and even though the step is a large geometric perturbation of the plane channel, it is not so large as to eliminate the pair structure of the eigenmodes and eigenvalues of the joint operator. This pairing of optimal disturbances, together with their orthogonality, implies some decoupling between the precisely defined step location and well-amplified disturbance flows in the channel many step-heights downstream. Physically, this is reasonable. In particular, it means that we can construct various initial conditions which give rise to similar amplifications and similar modes in the channel far downstream - the downstream modes differing essentially only by a phase shift of the rollers within a broader envelope.

We have noted the sharp concentration of optimal disturbance initial conditions around the step edge and that the associated structures extend upstream as well as downstream of this point. This implies that in order to capture fully the dynamics of convective instability in this flow, simulation domains must extend some distance upstream of the step edge, as well as having significant local refinement at this point. Our initial studies (with $L_{i}$ as small as one step-height) showed that maximum energy growths reduced as inflow length contracted. As we also noted previously, the domains required to study transient growth in this flow may require significantly longer outflow lengths than are required to study the asymptotic global instabilities.

\subsection{Related previous work for the backward-facing step and similar flows}

Marquet et al. (2006) examined optimal growth at $R e=800$ for a rounded backward-facing step geometry, constructed so as to avoid both an upper separation bubble and a salient step edge; the step height is the same as the upstream channel 
depth, but further downstream the geometry contracts so that ultimately there is no expansion. In some respects, our findings at $R e=500$ are similar to theirs, but there are also significant differences. In both studies, optimal disturbance initial conditions are concentrated at the step (or separation point), consisting of a small wave packet of transverse vortices that are inclined backward relative to the mean shear and which gain energy as they are tilted upright into an array of rollers. In both studies, the two- and three-dimensional optimal disturbances are similar in nature, the spanwise wavenumbers of the three-dimensional optima are of order unity, and the three-dimensional optimal energy growths are somewhat greater than for the two-dimensional cases. We also observe that the locations of the disturbances at $\tau_{\max }$ in both studies lie downstream of the last reattachment point, but in the work of Marquet et al. (2006) this centroidal location is of order eight step heights, as opposed to order $25 h$ in our geometry. A significant difference between the results of the two studies is the magnitude of maximum energy growth: Marquet et al. find $G_{\max } \approx 900$ at $R e=800$, whereas we have $G_{\max }=78.8 \times 10^{3}$, approximately two orders of magnitude greater, at the lower value of $R e=500$. (Also we note that $G_{\max }$ should increase by a substantial amount between $R e=500$ and 800 , see figure $11 \mathrm{~b}$.) At present, it is unclear if the much greater energy amplification predicted in our study stems from the presence of a sharp step edge, from a greater expansion ratio and the existence of an upper separation bubble in our case, or other factors.

A feature noted in experimental studies (Lee \& Sung 2001; Furuichi \& Kumada 2002 ) is the presence of flow oscillations with dimensionless centre frequency $f \sim 0.1$. In these studies, where Reynolds numbers are typically well above the onset of sustained turbulence, this frequency is generally associated with structures of good spanwise coherence, at least for downstream locations within the initial separation zone (see e.g. Lee \& Sung, figure 14; Furuichi \& Kumada, figure 11). At lower Reynolds number ( $R e=1050$ in our normalization), Kaiktsis et al. (1996) employed two-dimensional DNS with either initial or sustained perturbation to examine local convective instability in backward-facing-step flow with a nominal expansion ratio of two, and found flow oscillations of similar frequency content. For example, when the Poiseuille inflow was perturbed with Gaussian white noise, a narrow-band random vertical velocity response with centre frequency $f \simeq 0.1$ was observed at $x \simeq 28 \mathrm{~h}$ (Kaiktsis et al., figure 17). In $\$ 5.3$, we will return to this theme and demonstrate linkage between the frequencies and structures observed in continually perturbed flow and our predictions of optimal disturbances.

\subsection{Effect of inflow perturbation}

As an approximation to what might be observed in a physical experiment where the inflow contains some noise, we carry out DNS for $R e=500$ where the (Poiseuille) inflow is continually perturbed. We add time-varying pseudo-random zero-mean Gaussian white noise at standard deviation level $U_{\infty} / 100$ (the same level as chosen by Kaiktsis et al. 1996) in the crossflow velocity components, randomly uniform across the inlet, for both two-dimensional and three-dimensional simulations. In the three-dimensional case, as for the simulation described in $\$ 4.3 .2$, the spanwise wavelength is set at $L_{z}=2 \pi / 0.645=9.74$ (which corresponds to global maximum linear transient growth at $R e=500$ ), and 16 Fourier modes are employed. Note that these disturbances will excite all the Fourier modes in the simulation, both two-dimensional and three-dimensional. Although we have not studied this aspect in detail, a statistically steady state appears to be established at any streamwise location 
(a)

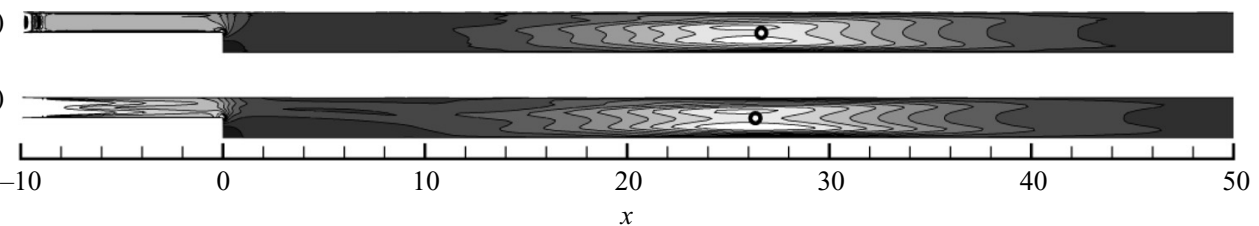

FIGURE 20. Contours of time-average perturbation energy obtained in DNS at $R e=500$ when the inflow velocity is perturbed crossflow by Gaussian noise with standard deviation level $U_{\infty} / 100$. Results for both $(a)$ two-dimensional and $(b)$ three-dimensional $(\beta=0.645)$ DNS are shown. Dots illustrate centroidal positions of energy distributions in the channels downstream of $x=2.5$; in each case these lie within 0.2 step heights of the location of the centroid of energy in the global optimal disturbance.

shortly after the inflow perturbation first arrives as it advects with the base flow. The results presented below were obtained after at least 500 time units had elapsed; with a mean advection speed of approximately $U_{\infty} / 3$, this is over three domain wash-through time scales. Statistics have been compiled for over 1000 time units.

Contours of time-average perturbation energy $\left\langle u^{\prime} u^{\prime}+v^{\prime} v^{\prime}+w^{\prime} w^{\prime}\right\rangle / 2$ for both a two-dimensional and three-dimensional $(\beta=0.645)$ DNS at $R e=500$ are shown in figure 20. In both cases, the perturbation energy is highest at the inflow boundary, then relaxes in the inflow channel. At the step edge, there is a sharp peak in perturbation energy, and immediately downstream of this the level drops. However, further downstream energy levels increase again to a maximum in the vicinity of $x=25$, before eventually falling. The behaviour downstream of the step is qualitatively similar to what would be expected of optimal linear transient energy growth.

One means of enabling a quantitative comparison is to compute the centroidal location of perturbation energy in the expanded channel. Making such a computation conditional on $x \geqslant 2.5$ (in order to remove any contribution of energy in the inflow channel and around the step), we find the centroid lies at $x_{c}=26.6$ in the twodimensional DNS and at $x_{c}=26.4$ in the three-dimensional case. A similar centroidal location of energy is observed in the two-dimensional global optimum perturbation for $R e=500$ at $\tau=58.0$ (figure $8 c$ ) which is $x_{c}=26.4$ and for the three-dimensional global optimum perturbation for $R e=500$ at $\tau_{\max }$ (figure $16 c$ ), which is $x_{c}=26.6$.

Figure $21(a)$ shows a time series of vertical velocity component extracted from the three-dimensional DNS at location $(x=25, y=0, z=0)$ after the simulation had settled to a statistically stationary state. The average velocity is slightly positive, matching that of the base flow at the same location. Although the inflow perturbations are white noise, the response at this location is clearly narrow-band. We note that the structure of this flow is relatively two-dimensional in structure in agreement with the location of the energy centroid in figure 20. Figure $21(b)$ shows the outcome of 10-pole maximum entropy spectral analysis, carried out after removal of the mean. The spectrum shows a single sharp peak with centre frequency $f=0.088$, virtually identical to what would be expected for wave packets of wavelength $L_{x}=3.73$ (cf. figure 17) advecting past at the average speed of Poiseuille flow in the downstream channel $\left(U_{\infty} / 3\right)$, i.e. $f=1 /(3 \times 3.73)=0.089$.

Figure 22 shows four snapshot contours of vertical $(y)$ perturbation velocity on the plane $y=0.25$, drawn from the same simulation as used for figure 21. Here the randomness of the inflow perturbation can be clearly seen near $x=-10$; this rapidly dies away, partly owing to the comparative coarseness of the mesh in this location, but 
(a)
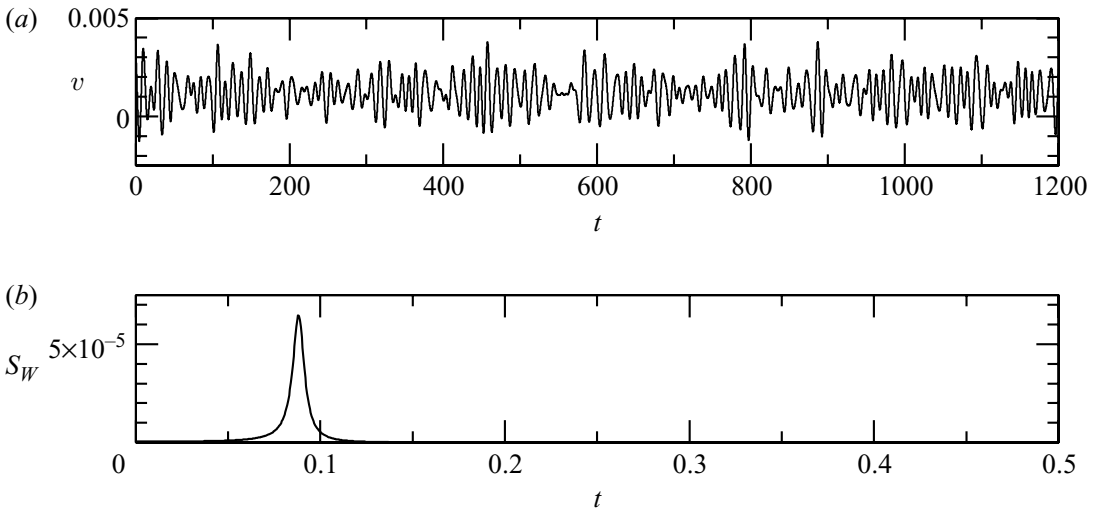

FIGURE 21. ( $a$ ) Time series of vertical velocity component at $(x=25, y=0, z=0)$, extracted from three-dimensional DNS at $R e=500, \beta=0.645$, where the Poiseuille inflow was continually perturbed with low-amplitude Gaussian white noise in the crossflow velocity components. $(b)$ Corresponding spectral density, centre frequency $f=0.088$.

(a)

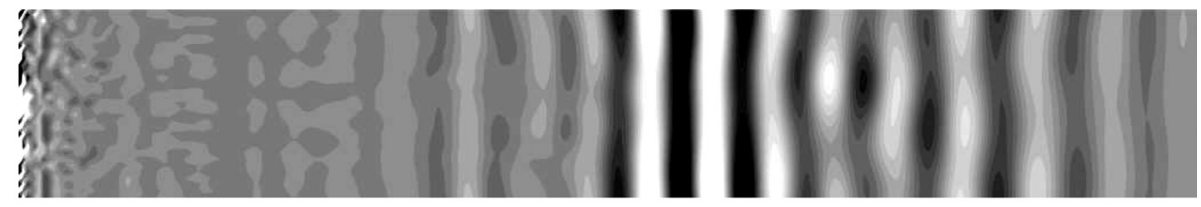

(b)

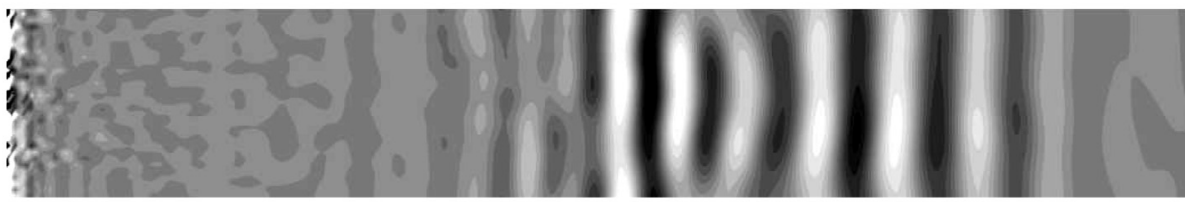

(c)

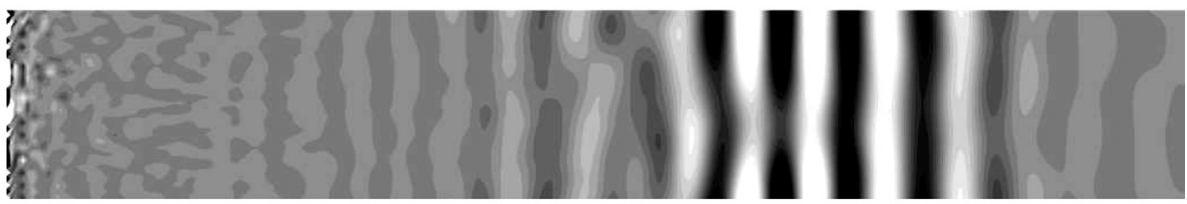

(d)

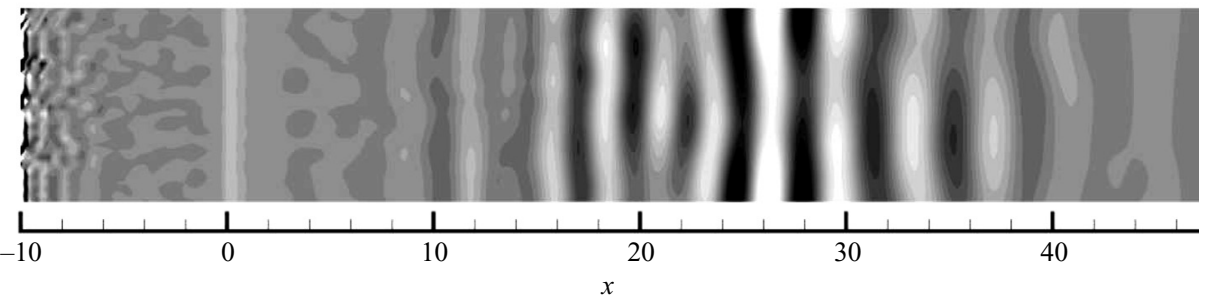

FIGURE 22. Contours of vertical perturbation velocity component on the plane $y=0.25$ at four instants $(t=300,600,900,1200)$ for the same simulation from which the data of figures $20(b)$ and 21 were obtained.

small-amplitude random fluctuations with little discernable structure may be observed up to $x=0$, i.e. the location of the step edge. For $x>0$, the perturbations form into predominantly two-dimensional wave packets, which reach maximum amplitude in the vicinity of $x=25$, before losing energy further downstream. 
(a)

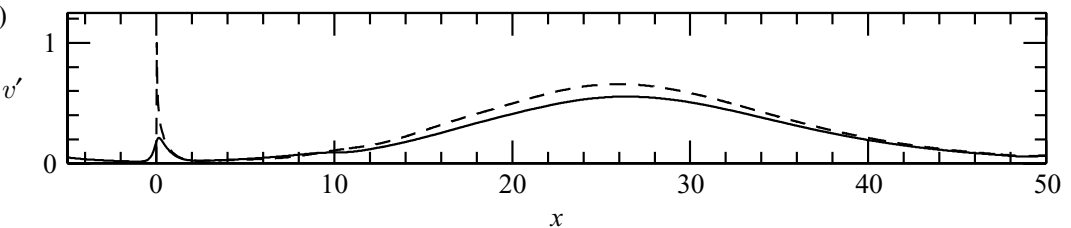

(b)

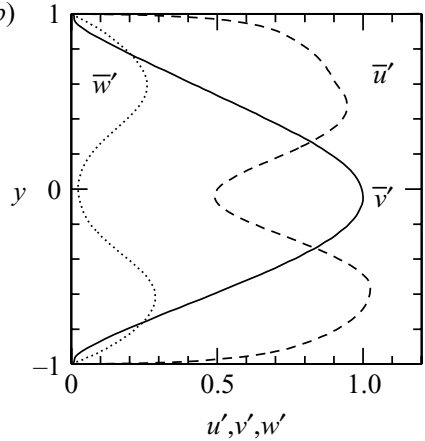

(c)

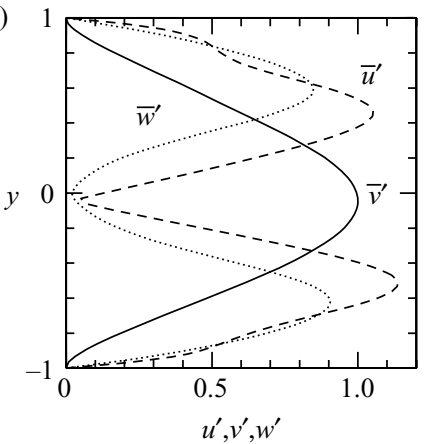

FIGURE 23. Profiles of fluctuating velocity components. (a) Vertical velocity standard deviation extracted along $y=0.25$ (solid line) and $y=0$ (dashed line), both normalized to the peak value for $y=0$. (b) Horizontal, vertical and spanwise velocity standard deviation profiles, normalized by peak value of vertical velocity standard deviation, extracted along line $x=26.5$. (c) Similarly normalized profiles of velocity component standard deviations in the optimal perturbation at $\tau_{\max }$ (i.e. figure $16 c$ ).

Figure 23(a,b) shows profiles of standard deviation velocity components for the simulation used to obtain the data of figure 21 . The values are all normalized by the maximum vertical velocity standard deviation. The profile of normalized vertical velocity standard deviation along the line $y=0$ (figure $23 a$ ) has a broad maximum centred at $x=26.4$, the same as the centroidal location of two-dimensional perturbation energy found for figure 20. Note also the much sharper peak of vertical velocity standard deviation immediately downstream of the step edge, which has a greater magnitude than that of the maximum further downstream. We take this to indicate that the flow at the step edge is receptive to perturbations at the domain inflow, but that not all of this response projects onto the optimal disturbance initial condition which subsequently leads to the downstream amplification. Figure $23(a)$ also shows a profile of vertical velocity standard deviation along the line $y=0.25$, corresponding to the elevation of data extraction used in figure 22. This profile still exhibits a peak near the step edge at $x=0$, but it is much less sharp, and of lower magnitude than for the corresponding peak in the profile obtained at $y=0$, showing that the high velocity fluctuations at the step edge are quite localized.

Profiles of normalized standard deviation velocity components $\left(\bar{u}^{\prime}, \bar{v}^{\prime}, \bar{w}^{\prime}\right.$ respectively for the $x, y$ and $z$ components) extracted along the line $x=26.5$ are shown in figure $23(b)$. The shape of the profiles - a peak on the channel centreline for the profile of $\bar{v}^{\prime}$, and two-lobed structures for the profiles of $\bar{u}^{\prime}$ and $\bar{w}^{\prime}$-are consistent with the physics of randomly excited wave packets of optimal disturbance type advecting past the extraction location. We demonstrate this by comparison with figure $23(c)$, which shows profiles of the velocity component standard deviations for the optimal disturbance at time $\tau_{\max }$ (see figure $16 c$ ). (Standard deviations were extracted along 
rays $y=$ const.) The greater relative amplitude of $\bar{w}^{\prime}$ in figure $16(c)$ compared with figure $16(b)$ is consistent with the fact that in the DNS, all Fourier modes (including the two-dimensional mode) are excited, weakening the relative contribution of the spanwise velocity component in figure $16(b)$.

These results for response to white-noise inflow perturbation in three-dimensional DNS are similar to findings reported by Kaiktsis et al. (1996) for two-dimensional flows; however, we have been able to demonstrate a more detailed linkage between optimal growth initial conditions and the downstream response, both in terms of temporal frequency and spatial structure. In simple terms, we can view the process as a convolution between perturbations present in the inflow and the system transient response as determined by the leading optimal growth initial conditions. Intermediate in this process is the receptivity of the flow at the step edge to inflow perturbations, and the projection of this response onto the optimal disturbance initial conditions for the base flow.

\section{Conclusions}

By employing timestepper-based methods for the computation of optimal flow disturbances in arbitrary geometries, we have confirmed that two-dimensional and three-dimensional flow past a backward-facing step supports large linear transient energy growth at Reynolds numbers for which the flow is asymptotically stable. In contrast with many past numerical studies of transient growth in shear flows, we have not focused on transition to turbulence brought about by perturbation. Rather, from the outset we have advocated such a transient growth study as the proper means to quantify convective instability in this flow, which does not undergo transition in the range of Reynolds numbers considered.

Three-dimensional disturbances of large spanwise wavelength are moderately favoured over two-dimensional disturbances, but the nature of the two- and three-dimensional disturbances is broadly similar. The optimal perturbation initial conditions at $R e=500$ consist of wavepackets of highly strained rollers that are inclined against the mean shear and are very tightly concentrated at the step edge. As time proceeds, these perturbations gain energy as they are tilted upright by the mean shear (the inviscid Orr mechanism), but also appear to interact with KelvinHelmholtz instabilities of the two separated shear layers, and maximum energy amplification occurs downstream of both separation bubbles. At this time, the optimal disturbance is a wavepacket of approximately elliptical rollers that fill the channel and have major axes that are aligned in the streamwise and crossflow directions. Their associated streamwise wavelength is approximately 3.73 step heights. At larger times, the disturbance dies away as the rollers advect downstream at the mean flow speed and are further strained in the direction of mean shear.

In attempting to relate the transient growth analysis to results that could be obtained in a physical experiment, we employ DNS in which the Poiseuille inflow is continually perturbed with zero-mean Gaussian white noise. Downstream of the step edge, we observe predominantly two-dimensional wave packets whose properties are related to the optimal disturbances.

H.M. B. wishes to acknowledge UK EPSRC grant EP/E006493/1 in financial support of a Visiting Fellowship, and support from the Australian Partnership for Advanced Computing. D. B. thanks the Ville de Paris for its support. S. J. S. would like to acknowledge financial support from an EPSRC Advanced Research Fellowship. 


\section{REFERENCES}

BARkLey, D. 2005 Confined three-dimensional stability analysis of the cylinder wake. Phys. Rev. E 71, 017301.

BARKLey, D. \& Henderson, R. D. 1996 Three-dimensional Floquet stability analysis of the wake of a circular cylinder. J. Fluid Mech. 322, 215-241.

Barkley, D., Gomes, M. G. M. \& Henderson, R. D. 2002 Three-dimensional instability in flow over a backward-facing step. J. Fluid Mech. 473, 167-190.

Barkley, D., Blackburn, H. M. \& Sherwin, S. J. 2008 Direct optimal growth analysis for timesteppers. Intl J.Numer. Meth. Fluids (in press).

Beaudoin, J. F., CAdot, O., Aider, J.-L. \& Wesfreid, J. E. 2004 Three-dimensional stationary flow over a backward-facing step. Eur. J. Mech. B/Fluids 23, 147-155.

Blackburn, H. M. 2002 Three-dimensional instability and state selection in an oscillatory axisymmetric swirling flow. Phys. Fluids 14 (11), 3983-3996.

Butler, K. M. \& FARrell, B. F. 1992 Three-dimensional optimal perturbations in viscous shear flow. Phys. Fluids A 4 (8), 1637-1650.

Chomaz, J., Huerre, P. \& Redekopp, L. 1990 The effect of nonlinearity and forcing on global modes. In New Trends in Nonlinear Dynamics and Pattern-Forming Phenomena (ed. P. Coullet \& P. Huerre), pp. 259-274. Plenum.

Chomaz, J.-M. 2005 Global instabilities in spatially developing flows: non-normality and nonlinearity. Annu. Rev. Fluid Mech. 37, 357-392.

Chun, K. B. \& Sung, H. J. 1996 Control of turbulent separated flow over a backward-facing step by local forcing. Exps. Fluids 21, 417-426.

Corbett, P. \& BotTARo, A. 2000 Optimal perturbations for boundary layers subject to stream-wise pressure gradient. Phys. Fluids 12 (1), 120-130.

Cossu, C. \& Chomaz, J. M. 1997 Global measures of local convective instabilities. Phys. Rev. Lett. 78, 4387-4390.

Ehrenstein, U. \& Gallaire, F. 2005 On two-dimensional temporal modes in spatially evolving open flows: the flat-plate boundary layer. J. Fluid Mech. 536, 209-218.

FARrell, B. F. 1988 Optimal excitation of perturbations in viscous shear flow. Phys. Fluids 31 (8), 2093-2102.

Furuichi, N. \& Kumada, M. 2002 An experimental study of a spanwise structure around a reattachment region of a two-dimensional backward-facing step. Exp. Fluids 32, 179-187.

Gresho, P. M., Gartling, D. K., Torczynski, J. R., Cliffe, K. A., Winters, K. H., Garratt, T. J., SPENCE, A. \& GoODRICH, J. W. 1993 Is the steady viscous incompressible two-dimensional flow over a backward-facing step at $R e=800$ stable? Intl J.Numer. Meth. Fluids 17, 501541.

GuERMOND, J. \& SHEN, J. 2003 Velocity-correction projection methods for incompressible flows. SIAM J. Numer. Anal. 41, 112-134.

Henderson, R. D. \& Barkley, D. 1996 Secondary instability in the wake of a circular cylinder. Phys. Fluids 8, 1683-1685.

HeppFner, J., Brandt, L. \& Henningson, D. S. 2005 Transient growth on boundary layer streaks. J. Fluid Mech. 537, 91-100.

Huerre, P. \& Monkewitz, P. A. 1985 Absolute and convective instabilities in shear layers. J. Fluid Mech. 159, 151-168.

Huerre, P. \& Monkewitz, P. A. 1990 Local and global instabilities in spatially developing flows. Annu. Rev. Fluid Mech. 22, 473-537.

Kaiktsis, L., Karniadakis, G. E. \& OrszaG, S. A. 1991 Onset of three-dimensionality, equilibria, and early transition in flow over a backward-facing step. J. Fluid Mech. 231, 501-528.

Kaiktsis, L., Karniadakis, G. E. \& OrsZaG, S. A. 1996 Unsteadiness and convective instabilities in two-dimensional flow over a backward-facing step. J. Fluid Mech. 321, 157-187.

Karniadakis, G. E. \& Sherwin, S. J. 2005 Spectral/hp Element Methods for Computational Fluid Dynamics, 2nd edn. Oxford University Press.

Karniadakis, G. E., Israeli, M. \& OrsZaG, S. A. 1991 High-order splitting methods for the incompressible Navier-Stokes equations. J. Comput. Phys. 97, 414-443.

LE, H., MoIN, P. \& KIM, J. 1997 Direct numerical simulation of turbulent flow over a backward-facing step. J. Fluid Mech. 330, 349-374. 
LEE, I. \& SUNG, H. 2001 Characteristics of wall pressure fluctuations in separated flows over a backward-facing step. Part I: Time-mean statistics and cross-spectral analyses. Exps. Fluids 30, 262-272.

Lien, F. S. \& LesChZINER, M. A. 1994 Assessment of turbulence-transport models including non-linear RNG eddy-viscosity formulation and second-moment closure for flow over a backward-facing step. Computers Fluids 23, 983-1004.

LiNDZEN, R. S. 1988 Instability of plane parallel shear-flow (toward a mechanistic picture of how it works). Pure Appl. Geophys. 126, 103-121.

LuCHINI, P. 2000 Reynolds-number-independent instability of the boundary layer over a flat surface: optimal perturbations. J. Fluid Mech. 404, 289-309.

MARQUET, O., SiPP, D. \& JACQUIN, L. 2006 Global optimal perturbations in a separated flow over a backward-rounded-step. In 36th AIAA Fluid Dyn. Conf. and Exhibit. San Francisco, paper. 2006-2879.

ORR, W. M. F. 1907 The stability or instability of the steady motions of a perfect liquid and of a viscous liquid. Part I: A perfect liquid. Part II: A viscous liquid. Proc. R. Irish Acad. A 27, $9-138$.

Schatz, M., Barkley, D. \& Swinney, H. 1995 Instabilities in spatially periodic channel flow. Phys. Fluids 7, 344-358.

Schmid, P. J. 2007 Nonmodal stability theory. Annu. Rev. Fluid Mech. 39, 129-162.

Schmid, P. J. \& Henningson, D. S. 2001 Stability and Transition in Shear Flows. Springer.

Sherwin, S. J. \& Blackburn, H. M. 2005 Three-dimensional instabilities and transition of steady and pulsatile flows in an axisymmetric stenotic tube. J. Fluid Mech. 533, 297-327.

Trefethen, L. N., Trefethen, A. E., Reddy, S. C. \& Driscoll, T. A. 1993 Hydrodynamic stability without eigenvalues. Science 261, 578-584.

Tuckerman, L. S. \& BARKley, D. 2000 Bifurcation analysis for timesteppers. In Numerical Methods for Bifurcation Problems and Large-Scale Dynamical Systems (ed. E. Doedel \& L. S. Tuckerman), pp. 453-566. Springer. 